Artikulation haben können, auch wenn sie weiterhin eine Wortassoziation ( $\mathrm{Rc}$ ) hervorrufen. Andererseits können wohl gelegentliche reine Wortassoziationen $(\mathrm{R}$ a) Formen des Versprechens erzeugen; sie sind jedoch schon für das sprechende Individuum vorübergehend und sind vollends für die übrigen Individuen einer Sprachgemeinschaft ohne Wirkung, da ihnen das Merkmal der 'Geläufigkeit' fehlt; nur wenn gleiche Assoziationen 'mitklingen', also bei 'geläufigen' Assoziationen, wird eine individuelle Analogiebildung(Form des Versprechens) infolge jenes Mitklingens Aussicht haben, Eigentum einer Sprachgemeinschaft zu werden. Wie sich der Übergang von der individuellen und okkasionellen zur usuellen Analogiebildung vollzieht, darüber wage ich vorläufig noch keine Vermutung: es ist ebensogut möglich, daß der Vorgang bei einem Einzelindividuum beginnt und sukzessive sich ausdehnt, wie daß spontan eine Mehrheit von Individuen die gleiche Analogiebildung schafft.

Marburg i. $\mathrm{H}$.

Albert Thumb.

\title{
Untersuchungen zur indogermanischen Altertumskunde.
}

Während auf dem Gebiet der indogermanischen Grammatik ein reges Leben herrscht und eine große Anzahl von Forschern an der Lösung der Probleme beteiligt sind, beschäftigen sich nur wenige mit der indogermanischen Altertumskunde, vielmehr ist diese seit Jahren fast ein Monopol von O. Schrader gewesen. Es ist nun niemals gut, wenn alle Arbeit im wesentlichen auf den Schultern eines Mannes ruht, denn jeder Mensch ist unvollkommen und er sieht die Dinge immer nur von einer Seite an. Wenn man bedenkt, wie die grammatischen Probleme hin- und hergewendet werden, ehe wir zu festen Ergebnissen kommen, wenn man sieht, wie langsam sich die richtige Erkenntnis hier Bahn bricht, so wird man es wohl für wünschenswert halten, wenn auch die Probleme der indogermanischen Altertumskunde einmal unter das Kreuzfeuer der Kritik genommen werden. Das hat ja P. v. Bradke getan, leider ist er aber der Wissenschaft allzu früh entrissen worden. Seine Stärke bestand in der Kritik, aber Kritik ist auf unserm Gebiet und vor allem gegenüber den Arbeiten 0. Schraders dringend nötig. P. v. Bradke hat sein 
Buch "Über Methode und Ergebnisse der arischen (indogermanischen) Altertumswissenschaft”, Gießen 1890, der Kritik der ersten Auflage von 0. Schraders Sprachvergleichung und Urgeschichte gewidmet, und wenn auch Bradkes Buch nicht gerade geschickt und glücklich geschrieben ist, sachlich hat er in fast allen Punkten recht. Er will im wesentlichen ja nur die Frage erörtern (S. 1), "unter welchen Bedingungen wir von der Ety mologie Auskunft über die Kultur der arischen Urzeit erwarten dürfen, was sich für diese aus sprachlichen Gleichungen ergibt und ob und wie weit Ergebnisse dieser Art fest genug stehen, um weitere Folgerungen tragen zu können". In der Tat hat v. Bradke gerade diese Seite erörtert, und wenn das Ergebnis des Buches im wesentlichen negativ ist, wenn es sich zeigt, daß sich aus den sprachlichen Tatsachen recht wenig ergibt, so lag darin ein Ergebnis vor, das später Kretschmer und Kossinna ihrerseits hervorgehoben haben. Auch ich habe durch Bradke gelernt, daß die Folgerungen aus der Sprache nur mit großer Vorsicht zu ziehen sind.

Die zweite Auflage von Schraders Werk hat v. Bradke in den Gött. Gel. Anz. 1890, 897 ff. besprochen, durchaus sachlich und gerecht; er erkennt an, daß in dem Buche manche Fortschritte zu verzeichnen sind, faßt aber sein Urteil in folgenden Worten zusammen: "Wenn der Herr Verf. fortfährt, sein Buch in der Richtung solcher 'Angriffe' wie des meinigen, die er als besonders unbegründet 'gelegentlich auch einmal zurückweisen zu sollen glaubt', fleißig umzuändern, so würde ich es nicht mehr für ausgeschlossen halten, daß es etwa in 4 . oder 5 . Auflage von den gröbsten Fehlern ziemlich frei wäre; nur müßte er mit der Aufnahme neuen Stoffes vorsichtiger werden". Das Urteil v. Bradkes wiegt nun sicher ungleich schwerer als das vieler anderer Forscher, die doch schließlich den behandelten Problemen ferner stehen, und es ist wohl an der Zeit, dieses Urteil wieder einmal anzuführen.

Jetzt erscheint Schraders Werk in dritter Auflage, und es ist daher dringend geboten, eine Reihe von Problemen neu zu erörtern, da auch in der neuen Auflage ein wesentlicher Fortschritt nicht $\mathrm{zu}$ verzeichnen ist.

Unterdessen hat 0 . Schrader ein neues großes Werk veröffentilichi, das Reallexikon der indogermanischen Altertumskunde. Ich habe dies Werk IF. Anz. 13, 5 ff. angezeigt und dabei versprochen, darauf zurückzukommen. Wenn dies bis jetzt noch nicht 
geschehen ist, so lag das an dem Mangel an Zeit, aber wenn ich auch spät komme, so komme ich doch, und die folgenden Aufsätze werden sich vielfach mit Schraders Werken und Auffassungen beschäftigen müssen.

Ich habe mich seit meiner Studentenzeit mit den kulturhistorischen Problemen, die die europäische Urzeit betreffen, beschäftigt, ich habe mein erstes Kolleg über die Urgeschichte der Indogermanen gelesen und dies in regelmäßigem Turnus bis jetzt wiederholt. Ich habe natürlich meine ersten Kenntnisse aus Hehn geschöpft und bin durch v. Bradke auf die Bedeutung Schraders hingewiesen worden. Schon im Jahre 1891 erschien mir Schraders Sprachvergleichung und Urgeschichte als ein unzureichendes Werk, und ich faßte damals den Plan, meinerseits ein anderes Werk zu schreiben, das nun endlich vollendet vorliegt. Auch dieses Werk erfordert noch einige Ergänzungen, es muß manches, was dort nur angedeutet wurde, näher begründet werden, und ich hoffe, daß sich auch andere Mitforscher an der Erörterung beteiligen werden. Im Verlauf der Zeit gedenke ich also an dieser Stelle eine Reihe von Aufsätzen zu veröffentlichen, die sich mit der indogermanischen Altertumskunde befassen.

\section{Wann können wir ein Wort für indogermanisch ansehen?}

Will man den Wortschatz der indogermanischen Ursprache für kulturhistorische Schlüsse verwenden, so muß doch zunächst die Frage entschieden werden, wann wir ein Wort für indogermanisch ansehen können. Der idealste Zustand ist es natürlich, wenn ein Wort noch in allen Sprachen erhalten ist, aber dieser Fall ist recht selten, und es ist ganz sicher, daß schon Worte, die nur noch in drei oder vier Sprachen vorliegen, für die idg. Ursprache in Anspruch zu nehmen sind. So lange eine bestimmte Stammbaumtheorie gebilligt wurde, war die Sache verhältnismäßig einfach. War ein Wort in je einer Sprache einer Gruppe belegt, so hatte man eigentlich die volle Gewähr für die Herkunft aus der Urzeit. Aber mit dieser Stammbaumtheorie steht es ja vorläufig schlecht, und man wird auf sie nicht bauen können. Schrader hat sich nun ein eigentümliches System zurecht gemacht. Sprachvergleichung und Urgeschichte ${ }^{3}$ S. 174 sagt er:

"Mir scheint die Sache so zu stehen, daß wenn ein Wort wenigstens in einer arischen und in einer europäischen oder wenigstens in einer nord- und in einer südeuropäischen oder 
wenn es auch nur im Griechischen und Lateinischen nachgewiesen werden kann, darin eine Garantie seines hohen Alters liegt." "Diese Auffassung," heißt es in der Anmerkung, "habe ich schon in der Vorrede zu meinem Reallexikon p. XIII deutlich ausgesprochen und bin ihr in meinem Buch gefolgt. Es ist daher nicht meine Schuld, wenn H. Hirt nicht hat entdecken können, welche Grundsätze mich bei dem Gebrauch des Wortes indogermanisch' geleitet haben." Ein anderes sind offenbar Grundsätze, und ein anderes ist es, ob man sie befolgt. Wir müssen also einerseits die Frage beantworten, ob diese Grundsätze richtig sind, und anderseits, ob 0 . Schrader sie befolgt hat. Daß letzteres nicht der Fall ist, läßt sich leicht zeigen, und da Schrader die Grundsätze schon in seinem Reallexikon befolgt haben will, so wählen wir die Beispiele daraus.

Zunächst haben doch die RL. S. 8 zusammengestellten Ausdrücke für Ackerbau, da auf sie alle die Bedingungen zutreffen, die Schrader aufstellt, nämlich daß sie in einer nord- und einer südeuropäischen Sprache oder im Griechischen und Lateinischen auftreten, ein volles Recht für indogermanisch zu gelten. Man kann also daraus nichts anderes schließen, als daß die Indogermanen den Ackerbau gekannt haben. Aber diese Ausdrücke sind nach Schrader europäisch-indogermanisch, womit ein neuer Begriff eingeführt oder vielmehr ein alter beibehalten wird. Aber wir haben ja auch eine nicht unbeträchtliche Zahl arisch-europäischer Ausdrücke, wie Schrader S. 10 hervorhebt, z. B. ai. yáva-, griech.

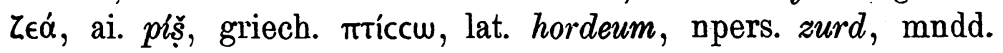
terwe, ai. dúrvā. Hier wird aber wieder von geringerer geographischer Verbreitung gesprochen. Was hat aber das mit unserer Frage zu tun, da die erwähnten Ausdrücke, da sie in getrennten Sprachen Asiens und Europas rorkommen, nach Schrader indogermanisch sind.

Anderseits erk]ärt Schrader folgende Gleichungen für indogermanisch: unter Feuer: got. fön, altn. fune, altpr. panno, also eine nur germ.-preußische Gleichung. Wels und altpr. kalis, d. lachs, slav. lososi haben dann doch dasselbe Anrecht. Vergl. darüber übrigens Schraders Reallexikon S. 495. Während die Gleichung $\pi \hat{\rho} \rho$, umbr. pir, ahd. fiur, armen. hur indogermanisch ist (S. 239), ist Te乇́kn, altpr. peuse, lit. puszis, ahd. fiuhto, ir. ochtach nur europäisch. Ich habe mich vergebens bemüht, hier Grundsätze zu entdecken. $\mathrm{Ob}$ für die Milch ein indogerm. Ausdruck vor- 
handen war, sagt Schrader nicht ausdrücklich. Wir finden bekanntlich verschiedene Ausdrücke, "die sich merkwürdigerweise immer auf zwei Sprachen beschränken: ai. dadhan, apr. dadan; griech. $\gamma \alpha \dot{\alpha} \lambda \alpha$, lat. lac; got. miluks = ir. melg”. Ich glaube, auch Schrader wird diese Ausdrücke für indogermanisch halten. Aber dann haben doch got. gulp, abg. zlato, lett. zelts, die sogar Ablaut zeigen, griech. $\chi \alpha \lambda \kappa o ́ c$, abg. żelèzo dasselbe Anrecht, für die Ursprache in Anspruch genommen zu werden.

Ich könnte noch mehr Beispiele anführen, aber sie würden nur beweisen, daß es etwas anderes ist, Grundsätze aufzustellen, und etwas anderes, sie zu befolgen. Wenn man aber alle derartigen Fälle zusammenhält, so berechtigen sio wohl zu dem Urteil, daß man nicht imstande ist, Schraders Grundsätze zu ermitteln. Schraders Vorgehen ist aber trotz des Mangels an irgend welchen leitenden Prinzipien ziemlich klar. Er hat sich seit geraumer Zeit bestimmte Ansichten über die Kultur der Indogermanen gebildet, Ansichten, die z. T. auf V. Hehn zurückgehen, und nach diesen Ansichten werden die Tatsachen, ich kann nicht anders sagen, gepreßt. Weil die Indogermanen nach Schrader Nomaden waren, darum sind die Ackerbauausdrücke nicht indogermanisch, weil sie in der Steppe wohnten, darum können es die Baumnamen ebensowenig sein. Damit wird aber unsere ganze Wissenschaft hinfällig. Ich habe in meinen Indogermanen schon hervorgehoben, daß wenn man die Ausdrücke, die sich auf die Viehzucht beziehen, mit demselben Maß mißt, wie die Ackerbauausdrücke, man dann auch dazu kommen kann, den Indogermanen die Viehzucht abzusprechen.

Wenden wir uns nun zu den Grundsätzen selbst. Schrader erkennt also im Prinzip idg. Gleichungen an, die nur in je zwei Sprachen belegt sind. Das ist in der Tat richtig. Man wird hinzufügen können, daß gewiß viele Worte des Indogermanischen überhaupt verloren gegangen sind, andere sich nur in einer Sprache erhalten haben. Daß wir mit dieser Tatsache rechnen müssen, ergibt sich aus den Parallelen, die die moderne Entwicklung bietet. Nicht wenige Worte, die sich in den altgermanischen Zeiten finden, sind in den modernen Dialekten gänzlich ausgestorben. Liegt mhd. geswio, agerm. ehu 'Pferd' noch irgend wo vor? und wenn sie irgendwo belegt sein sollten, wie könnten wir ihr hohes Alter erhärten, wenn wir nicht die alten Quellen hätten? Sollen die Slaven etwa den alten idg. Ausdruck patér nie besessen 
haben? Ein Romanist kann gewiß viele lat. Worte nachweisen, die im Romanischen völlig ausgestorben sind oder sich vielleicht nur in einer einzigen Sprache erhalten haben. Mit diesen völlig verlorenen Worten können wir natürlich nichts anfangen, wohl aber müssen wir unsere Aufmerksamkeit auf die isolierten Worte der Einzelsprachen richten.

Wir haben natürlich in den Fällen, wo ein Wort einer Einzelsprache in den verwandten Dialekten nicht wiederkehrt, nur geringen Anhalt, um das höhere Alter des Wortes zu erhärten. Aber wir haben doch manchmal einen Anhalt. So kann man annehmen, daß germ. hand ein indogerm. Wort ist, erstens weil man an einen Zusammenhang mit dem Zahlwort zehn, idg. dek'mt denken kann, und zweitens weil es ein konsonantischer Stamm ist. Neue konsonantische Stämme sind aber im Germanischen kaum noch gebildet worden, denn wir haben es hier mit ganz geringen Resten einer einst weiter verbreiteten Flexion zu tun, die wohl schon im Indogerm. unproduktiv geworden ist. Kahle "Zur Entwicklung der konsonantischen Stämme im Germanischen" verzeichnet die Stämme, die nach der konsonantischen Deklination im Germanischen gehen, und von diesen sind folgende ganz sicher indogermanisch : Fuß, Zahn, Monat, Maus, Gans, Nacht, Tür, Kuh. Bei den andern spricht jedenfalls nichts dagegen, es sind Winter, Genosse, Magd, Hand, Brust, Burg, Buch, Bruch, Eiche, wenn sie auch in andern Sprachen nicht belegt sind. Bei hand ist gegen die Ableitungen von got. hinpan 'fangen' semasiologisch nichts einzuwenden, wohl aber von Seiten der Form. Im Germanischen kann das Wort nicht erst abgeleitet sein, weil jede Analogie fehlt.

Wir haben also in der Form ein Hilfsmittel, das Alter eines Wortes zu bestimmen, und wer dieser Frage einmal systematisch nachgeht, der wird zu ganz interessanten Ergebnissen kommen. Man nehme einmal die Worte für König. Indogermanisch ist rēks, ai. rājă lat. rēx, kelt. rīx. Die sonstigen Ausdrücke der Einzelsprachen sind aber meist ganz deutliche Ableitungen, und es steht der Annahme nichts im Wege, daß sie erst im Leben der Einzelsprache neu gebildet sind, wenn sie auch z. T. nicht neu gebildet sein müssen. So z. B. ahd. kuning, zu kuni 'Geschlecht', got. piudans zu piuda 'Volk', ahd. truhtīn zu truht 'Schar', got. kindins: lat. gens. Mit diesen durchsichtigen Bildungen vergleiche man einmal die germanischen 
Ausdrücke für die See und die Seelandschaft. Zwar kehren sie in den verwandten Sprachen nur selten wieder, aber sie zeigen ein durchaus altertümliches Gepräge. Meistens mangelt daher auch jede Etymologie.

Dahin gehören got. saiws, d. See (die Beziehung zu lat. saevus ist doch nur ein Notbehelf. Wie will man den $i$-Stamm erklären?), altn. haf, ags. hoef, d. Haff (ist ebenso unklar), got. flödus, ahd. fluot (sieht durchaus alt aus. Ebenso) got. wëgs, ahd. $w \bar{a} c$ 'Woge' (woher der i-Stamm ?), altn. klif, ahd. clëp 'Klippe', altn. sund, ags. sund, d. Riff, Geest usw. Bei allen diesen Worten ist der Verdacht, daß sie viel älter sind als die germanische Sonderentwicklung durchaus berechtigt.

Da die Vriddhibildungen kaum anderswo als im Indischen produktiv geworden sind, so haben Bildungen dieser Art, die nur in einer Sprache vorliegen, die Gewähr hohen Alters. Wer dies bedenkt, wird an dem hohen Alter von ahd. swägur kaum zweifeln. Entsprechend wird man für ahd. huon indogermanisches Alter in Anspruch nehmen. Sachlich hat das gar keine Schwierigkeiten, da Hahn und Huhn nicht nur das Haushuhn bezeichnen, sondern in der Jägersprache auch das Rebhuhn, sowie den männlichen und weiblichen Vogel überhaupt.

Man muß sich also die Worte, die nur in einer Sprache belegt sind, auf ihre Bildung ansehen, ehe man sie für eine spezifische Neubildung erklärt, ja man kann sogar sagen, was nicht als deutliche Ableitung in einer Sprache erkennbar ist, ist höhern Alters sehr verdächtig. - Größere Sicherheit für Herkunft aus der indog. Ursprache erlangen wir, wenn ein Wort aus zwei Sprachen belegt ist. Hier schließt nun aber Schrader gewisse Sprachgruppen aus, nämlich etwa keltischgermanische und germanisch-lituslavische, aber auch keltischslavische Gleichungen, falls er nicht etwa keltisch zum Südeuropäischen rechnet. In dieser Ansicht haben wir offenbar eine Nachwirkung der Schmidtschen Wellentheorie. Ob diese begründet ist, will ich hier nicht untersuchen, sondern später noch einmal darüber sprechen. - Die erwähnten Sprachgruppen sind einander in historischer Zeit benachbart, und es liegt daher der Verdacht nahe, daß die eine von der andern Wörter entlehnt hat; wie wir wissen, haben die Germanen von den Kelten, die Slaven von den Germanen empfangen. Handelt es sich nun um Worte, bei denen der Verdacht der Entlehnung nicht aus- 
zuschließen ist, so wird man sie besser nicht zu Rückschlüssen auf die ältere Zeit verwenden, bei allen andern steht dem aber nichts im Wege. Ich sehe durchaus keinen Grund, Gleichungen wie d. lahs, lit. lašišà, russ. lososi, got. gulp, abg. zlato nicht gelten zu lassen. Auch an d. Stute, slav. stado kann man keinen Anstoß nehmen, sowie an anderen Gleichungen, die sich auf zwei nordeuropäische Sprachen beschränken. Wenn man sich einmal klar gemacht hat, wo die Germanen und die Slaven ursprünglich gesessen haben, die einen in Schleswig-Holstein usw., die anderen hinter den Karpathen, so wird man kein Bedenken tragen, Gleichungen, die in diesen beiden Sprachgruppen auftreten, zu verwenden. Wer dagegen ist, müßte nachweisen, daß die Germanen und Slaven eine Zeit der gemeinsamen Sonderentwicklung durchgemacht haben. Ich will die Beweise hierfür abwarten, vorläufig aber beharre ich auf meiner Ansicht, daß zwischen Slavisch und Germanisch keine besondern Berührungen vorhanden sind, und daß wir demnach Worte, die nur in diesen beiden Gruppen auftreten, sehr wohl für die Erschließung der indogermanischen Kultur verwenden können. Man kann ja auch den Gegenbeweis antreten. Welche germanisch-slavische Gleichung dürfen wir nicht der indogermanischen Ursprache zuschreiben, weil etwa kulturhistorische Erwägungen allgemeiner Art dagegen sprechen? Ich bin auf den Nachweis einer solchen Gleichung gespannt.

Und ebenso, wie mit den slavisch-germanischen steht es mit den keltisch-germanischen. Zwar sieht J. Schmidt im Keltischen sozusagen das Mittelglied zwischen Italisch und Germanisch, aber er stützt sich nur auf die Argumente Ebels; diese sind indessen kaum haltbar, und neue sind nicht beigebracht worden. Ich habe mich bemüht, neue Gründe beizubringen. Aber weder bei Kluge Pauls Grd. 1, 325, noch bei Bremer ebd..2 3, 27 ist irgend etwas verzeichnet. Die Frage bedarf erneuter Untersuchungen, sagen beide Forscher. Ich habe mich an Thurneysen um Auskunft gewendet, aber auch er konnte nichts mitteilen, was für nähere Beziehungen des Keltischen und Germanischen wesentlich in die Wagschale fiele. Ich bin daher auf den Gedanken gekommen, daß die Nachbarschaft der Kelten und Germaner verhältnismäßig jung ist, und daß daher auch keltischgermanische Gleichungen unbedenklich zu verwenden sind, unter Beachtung der oben herrorgehobenen Kautelen. 
Während wir bei den etymologischen Untersuchungen und Vergleichungen häufig mit Wurzeln operieren, können uns diese, wenn wir Kulturgeschichte treiben, wenig nützen. In diesem Fall bedürfen wir der vergleichbaren, möglichst genau stimmenden Worte. Wenn man einmal ein Wörterbuch derartiger Worte zusammenstellte, so würde man sehen, wie klein der erschließbare Wortschatz des Indogermanischen noch immer ist. Er würde so klein ausfallen, daß man sich sofort sagen würde, das kann nicht alles sein, da muß vieles verloren gegangen sein, und wir könnten schon deshalb nicht auf die Gleichungen, die nur in zwei Sprachen belegt sind, verzichten.

Und selbst kelto-italische oder indo-iranische Gleichungen sind wertvoll; denn mag man so niedrig rechnen wie man will, um 1200 müssen sich doch wohl die Italiker von den Kelten getrennt haben, und man wird für die Trennung der Inder und Iranier vielleicht noch zu einer früheren Zeit kommen. Da es die Aufgabe der indogermanischen Altertumskunde ist, die prähistorische Zeit aufzuhellen, so sind auch Gleichungen, die nur diesen Sprachen angehören, wichtig. Unsere Hilfsmittel sind viel zu dürftig, als daß wir auf irgend ein Moment verzichten dürften.

Jedenfalls glaube ich gezeigt zu haben, daß weder Schraders Grundsätze haltbar sind, noch daß er die von ihm aufgestellten Grundsätze befolgt, und daß sich meine Anschauungen sehr wesentlich ron den seinigen unterscheiden.

Haben wir nun die Wortformen festgestellt, so kommt die Frage nach der Bedeutung hinzu. Auch in diesem Punkt braucht man nicht allzu ängstlich zu seiu. Wir wissen, daß Bedeutungsübergänge gleicher Richtung oft genug an den verschiedensten Stellen vorkommen, aber, soweit meine Kenntnis reicht, ist der Fall außerordentlich selten, daß der gleiche Bedeutungsübergang bei demselben Wort eintritt. Der Fall also, den Hehn angeführt hat, daß Worte, die ursprünglich 'zerreiben' bedeutet haben, in die Bedeutung 'mahlen' übergehen, ist denkbar, unwahrscheinlich aber, daß das gerade selbständig bei demselben Worte molo eingetreten sein sollte? Gesetzt, die idg. Wurzel sëhabe 'werfen' bedeutet, so ist es nicht glaublich, daß sich im Ital., Kelt., Germ. und Lit.-Slav. überall erst in einzelsprachlicher Zeit die Bedeutung 's äen' sollte entwickelt haben.

Diese Frage bedarf aber noch weiterer Ausführungen, die ich auf eine spätere Zeit verschiebe. Nur das möchte ich noch hervorheben, daß schon A. Kuhn ein nach meiner Auffassung richtigeres 
Bild von der Kultur der Indogermanen entworfen hat als 0. Schrader, weil er sich eben nicht auf eine vorgefaßte Meinung stützte, sondern weil er sich an die Tatsachen der Sprache hielt.

\section{Läßt sich aus dem Fehlen von etymologischen Gleichungen für gewisse Begriffe etwas erschlieBen?}

Es ist ganz sicher, daß wir den Wortschatz der indogermanischen Ursprache niemals vollständig erschließen können, ebensowenig wie wir den lateinischen aus den romanischen Sprachen ganz rekonstruieren können. Nun tritt nicht selten der Fall ein, daß Worte für bestimmte Begriffe fehlen. So gibt es kein erschließbares Wort für 'Dampfschiff' oder 'Eisenbahn'. Solche Fälle wird aber wohl jeder ausschließen, da es sich um Dinge handelt, die nachweislich spät aufgekommen sind. Anders steht es, wenn Gleichungen für Dinge fehlen, die in der Urzeit vorhanden gewesen sein können. Im allgemeinen ist man zu der Erkenntnis gekommen, daß aus dem Fehlen von etymologischen Gleichungen nichts zu erschließen ist. Wenn wir keine Gleichungen für 'Löwe, Tiger, Kamel, Palme' antreffen, so beweist das nicht, daß die Urheimat in einem Gebiet lag, das diese Tiere nicht kannte; denn wenn die Urheimat diese Tiere besaß, die Indogermanen aber die Gegend verließen, so mußten, wenn die Tiere aus dem Gesichtskreis verschwanden, auch die Worte verloren gehen. Ähnlich ist das Wort 'Elch' den deutschen Dialekten verloren gegangen, weil das Tier aus dem größten Teil Deutschlands verschwunden ist, usw. Das erkennt Schrader Spr. u. U. ${ }^{3} 161$ auch an, er fügt aber S. 162 hinzu: "Nun soll aber damit keineswegs gesagt sein, daß dem Abhandensein urverwandter Gleichungen für die Erschließung der Urzeit jeglicher Wert abzusprechen sei. Im besonderen wird man nicht an ein zufälliges Aussterben einst vorhandener Ausdrücke denken dürfen, wenn es sich um ganze Begriffskategorieen handelt". Er sucht dies an den Fischnamen zu zeigen und fährt fort: "Ebenso bezeichnend wie die Armut einer urverwandten Terminologie auf dem eben erörterten Gebiete der Fischerei erscheint mir die gleiche Erscheinung auf dem der Schiffahrt gegenüber dem des Wagenbaus, dem der Blumenzucht gegenüber dem des Ackerbaus, dem der Verschwägerungsbezeichnungen des Mannes gegenüber denen des Weibes, auf dem Gebiet der Götternamen gegenüber dem der Personennamen usw." 
Ich stehe auf einem andern Standpunkt und habe in meinen Indogermanen und auch sonst den Satz ausgesprochen, daß sich aus dem Feblen von Worten nie etwas schließen läßt. Vielleicht ist der Satz in dieser Schärfe nicht ganz richtig, vergl. oben die Bemerkung über 'Dampfschiff' und 'Eisenbahn', aber ich kenne bisher keinen Fall, durch den er widerlegt würde. Jedenfalls müssen wir uns zunächst mit den Schraderschen Kategorien beschäftigen, um zu untersuchen, ob bei ihnen die Schlußfolgerungen des Autors wirklich zulässig sind.

\section{A. Der Mangel an Fischnamen.}

Es scheint eine fable convenue zu sein, daß es indogermanische Fischnamen nicht gibt. Ich vermute, daß sie im letzten Grunde darauf zurückgeht, daß Pictet Les origines indoeuropéennes keine Fischnamen verzeichnet ${ }^{1}$ ). Es wäre aber wirklich gut, wenn dieser Punkt endlich einmal aus der Erörterung verschwände.

Wir haben zunächst einen indogermanischen Ausdruck für Fisch; lit. žuvis, armen. jukn, griech. ix $\theta \hat{v} c$ sichern auch nach Schrader den Begriff für das Indogermanische. Aber auch lat. piscis, ir. iasc, got. fisks müßte doch nach demselben, da eine nord- und eine südeuropäische Sprache zeugt, indogermanisch sein. Wir haben ferner das Wort für Aal, lat. anguilla, griech.

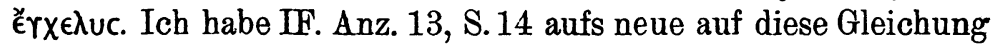
hingewiesen, worauf Schrader Sprachvergl. und Urgesch. ${ }^{3}$ S. 162 in der Anmerkung hervorhebt, "ich gäbe den gegenwärtigen Stand unseres Wissens unrichtig an, wenn ich die Verwandtschaft von

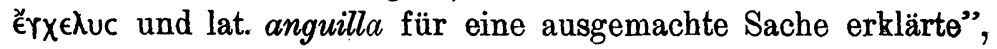
und er verweist dafür auf Waldes etymologisches Wörterbuch. Nun Walde in allen Ehren, aber ein Kronzeuge ist er in diesem Falle denn doch nicht. Hier muß die eigene Untersuchung einsetzen, nicht die des Verfassers eines lateinischen Wörterbuches - denn für ihn ist es verhältnismäßig gleichgiltig, ob anguilla

1) Ich benutze die Gelegenheit, um über Pictets Werke einiges zu sagen. Die Sammlung des Wortschatzes ist für die damalige Zeit wirklich allen Lobes wert, und in dieser Sammlung liegt noch heute die Bedeutung dieses Buches. Es ist doch wahrlich kein großes Verdienst, die unhaltbaren Gleichungen dieses Werkes zu erkennen und ein paar neue hinzuzutun. Daß der prinzipielle Standpunkt Pictets unhaltbar ist, wissen wir durch Hehn and nicht durch Schrader, diesem gegenüber bietet Pictet in vielen Punkten besseres, weil er die Tatsachen ruhig sprechen läßt. 


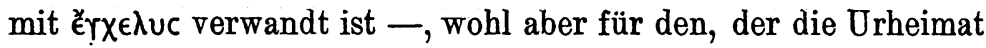
der Indogermanen bestimmen will und der solche Behauptungen über die Fischnamen aufstellt wie Schrader.

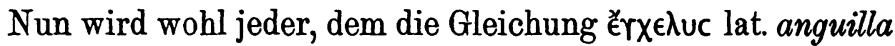
vorgelegt wird, zuerst an Urverwandtschaft denken; denn die Worte decken sich nicht nur im Stamm, sondern auch in dem merkwürdigen Suffix. Allerdings bleiben einige Schwierigkeiten. Die eine bildet die Verschiedenheit der Vokale. Hier hat uns J. Schmidts hochbedeutender Aufsatz KZ. 32, $321 \mathrm{ff}$. geholfen, und Schmidt hat denn auch schon bemerkt, daß der Gen. érxétuoc

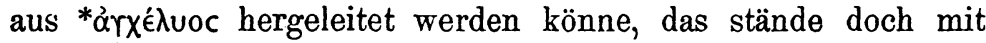
Étépou aus átépou ganz auf einer Linie. Die zweite wichtige Frage ist, konnte Ěr $\gamma \in \lambda \cup c$ im Griechischen, anguilla im Lat. mit den Suffixmitteln der Sprache neugebildet werden? Aber die Suffixform von Ë $\gamma \chi \in \lambda \cup c$ ist im Griechischen ganz vereinzelt, vgl. L. Meyer Handbuch der griech. Etym. 1, 425. Mit Suffix $l u$ kenne ich nur $\theta \hat{\eta} \lambda u c$. Außerdem ist der Stamm èrX- im Griechischen nicht be-

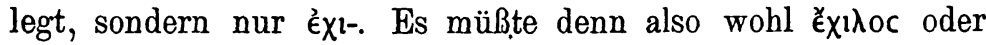
ĚXı $\lambda \cup c$ heißen. Es ist doch wirklich ein starkes Stück, ein Wort in einer Sprache für eine Neubildung zu halten, wenn weder der Stamm belegt, noch das Suffix produktivist. So lange diese Schwierigkeiten nicht gehoben sind, würde ich das griechische Wort nach den oben gegebenen Ausführungen für indogermanisch halten, selbst wenn es in keiner andern Sprache belegt wäre.

Fast ebenso schwierig ist auch die Annahme, daß lat. anguilla neugebildet sei. Allerdings haben wir hier das Grundwort anguis, aber das Suffix ist ebenfalls selten und nicht produktiv, abgesehen von den Fällen, wo es Diminutiva bildet und durch Assimilation aus $r l$ usw. entstanden ist. Aber schon Prisc. gramm. II 115, 13 bemerkt, anguis anguilla, unguis ungula, nubes nubilum, quae magis denominativa sunt existimanda quam diminutiva, quippe non habent diminutivorum significationem, sed formam tantum. Ich bestreite es nun entschieden, daß der Aal eine kleine Schlange ist, wenigstens in Europa sind kaum Schlangen vorhanden gewesen, denen gegenüber der Aal als klein erschienen wäre. Ich glaube daher nicht, daß -illa in anguilla das Diminutivsuffix ist.

Außerdem ist zu beachten, daß anguis ein Maskulinum war, weshalb ist also anguilla wie auch ér $\curlyvee \in \lambda \cup c$ meist im Griechischen Femininum, da doch auch piscis Maskulinum ist? Das sind doch alles ganz einfache Erwägungen, die jeder anstellen muß, der 
sich mit diesen Worten beschäftigt. Auch Thurneysen ist im

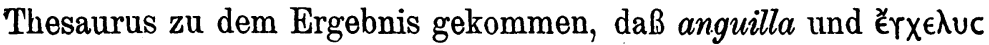
vielleicht zusammengehören.

Gefördert ist unser Problem schon längst durch einen Aufsatz von W. Meyer KZ. 28, 162 ff., in dem er S. 163 anguilla auf eine Flexion *anguilū, *anguiluās zurückführt, einer Bildung wie ai. vadhüşs, griech. $\pi \rho \epsilon ́ c \beta \alpha$. Diese Auffassung hat sich Johannsson KZ. 30, 425 angeeignet, wie ich glaube mit vollem Recht. Zunächst freilich ist noch die Frage zu erörtern, ob $l w$ zu $l l$ im Lat. assimiliert ist, was Brugmann Grdr. $I^{2} 325$ etwas zweifelhaft erscheint, während sich Stolz Lat. Gr. ${ }^{3}$ 88, Sommer Handbuch 226 dafür aussprechen. Ich muß mich ihnen durchaus anschließen trotz Solmsen KZ. 38, $437 \mathrm{ff}$. Ich halte an Gleichungen wie pallidus und lit. paivas, altbulg. plavi, ahd. falo, pollen zu pr. pelwo, abg. plèva 'Spreu' entschieden fest. Und anguilla fällt nicht minder in die Wagschale. Wie will man denn die Suffixgestalt erklären? Nehmen wir aber anguilua als Grundform an, so haben wir eine fast vollständige Übereinstimmung zwischen dem griechischen und lat. Wort 1). Es bleibt nur noch die Verschiedenheit der Gutturale. Aber hier kann entweder anguilla sein $u$ von anguis bekommen haben, oder Ě $r \chi \in \lambda \cup c$ hat sein $u$ im Griechischen verloren (wegen des folgenden $u$ ?), vielleicht schon im Indogermanischen. - Aber wir haben noch eine zweite Gleichung für

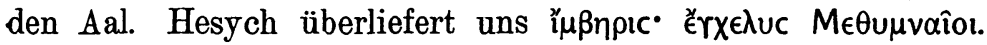
Das Wort steht an richtiger etymologischer Stelle, wir haben also keinen Grund es zu beanstanden. Diesem Wort entspricht im Lit. ungurỹs, russ. ugri. Die Gleichung ist auch tadellos, und es ist auch hier kaum denkbar, daß die Worte erst in den Einzelsprachen mit ihren Suffixmitteln gebildet seien.

Schließlich haben wir noch ein drittes Wort für 'Aal' in germ. Aal, das bisher unerklärt ist; denn E. Schröders Herleitung aus édlos 'der Fresser' (ZfdA. 42, 63) ist doch nur ein Notbehelf und unterliegt lautlichen wie semasiologischen Bedenken. Vielleicht läßt sich aber das Wort doch aufklären. Das griechische Wort Ër $\gamma \in \lambda u c$ kann vom Standpunkt des Griechischen, wie wir sahen, kaum neu gebildet sein. Aber auch vom Indogermanischen aus gesehen, d. h. wenn wir das Wort für indogermanisch halten,

1 Lat. helvos und germ. gelb, die Solmsen a. a. 0. ins Feld führt, können sich nicht genau entsprechen, da urital. helvos zu holvos hätte werden müssen, vgl. Sommer Hdb. S. 76. 
bereitet es große Schwierigkeiten, da wir offenbar zwei Vollstufen neben einander haben. Außerdem ist Suffix -lu außerordentlich selten und -elu ist ganz und gar nicht nachweisbar. Das Wort für Schlange idg. *angh ${ }^{\star} i s$, von dem man es abzuleiten versuchen könnte, ist deutlich $i$-Stamm, lat. anguis, lit. angis usw. So kommt man unwillkürlich auf den Gedanken, daß das Wort ein Kompositum ist argh $h^{w-e l u s, ~ u n d ~ d i e s e s ~ z w e i t e ~ E l e m e n t ~-e l u s ~ k o ̈ n n t e ~}$

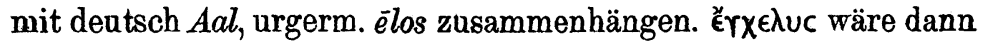
eine Bildung wie Adler aus adel-ar, und in él (Dehnstufe zu el) würde ich also die ursprüngliche Bezeichnung des Aales sehen. Was dies eigentlich bedeutete, können wir nicht wissen. $\mathrm{Ob}$ es sich bei der Bildung des Wortes um verschiedene Suffixgestalt. handelt, oder ob $w$ nach $l$ im Indogermanischen unter besonderen Umständen schon geschwunden ist, läßt sich kaum sagen. Jedenfalls gibt es Mittel genug, um aus diesen Schwierigkeiten herauszukommen. Den Stamm $e l$ haben wir im Deutschen noch in einem Fischnamen, in ahd. alunt, anord. olunn, dessen Stamm man schon längst mit dem von Aal verglichen hat. Äußerlich haben die beiden Fische freilich wenig gemein. Aber auch wenn meine Erklärung des Wortes Aal nicht richtig wäre, so würde ich in ihm doch immer einen indogermanischen Fischnamen ansehen, weil er aus germanischem Sprachgut nicht zu erklären ist ${ }^{1}$ ). Eine ähnliche Kompositionsbildung für Aal haben wir auch in ir. esc-ung; dessen letzten Bestandteil man vielleicht mit lat. anguis zusammenbringen kann. Ob auch der zweite Teil von griech. i $\mu \beta-\eta \rho ı c$ ein selbständiges Wort ist, weiß ich nicht zu sagen.

Jedenfalls sieht man, welche Bewandtnis es mit Schraders Behauptung hat, daß es keine gemeinsamen indogermanischen Fischnamen gäbe ${ }^{2}$ ). Außerdem gibt es aber noch andere Fisch-

1) Schrader will seine verlorene Position, dả die Indogermanen am Schwarzen Meer saßen, dadurch retten, daß er ein Gutachten veroffentlichen will, nach dem der Aal von jeher im Schwarzen Meer vorgekommen wäre. Gut, wenn es gelingt. Ich konnte diese Untersuchungen nicht kennen, konnte mich vielmehr nur auf die verbreitete Ansicht der Zoologen stützen, daß der Aal im Schwarzen Meer nicht vorkommt. Im übrigen wird jeder aus der Darstellung in meinen Indogermanen' ersehen, wie wenig für mich diese tiergeographischen Argumente ins Gewicht fallen. Weshalb Schrader nicht versteht, wie ein gemeinslavischer Name des Aales vorhanden sein kann, weiß ich nicht, vielleicht belehren ihn aber die Karten bei Niederle Slovanskê Starožitnosti.

2) $\mathrm{DaB}$ der Aal bei Homer nicht zu den Fischen gerechnet wird, kann nicht besonders auffallen. 
namen. Ahd. lahs, russ. lososi, lit. lašišà ist eine Gleichung, der so gut indogerm. Alter zukommt wie jeder andern; denn das Wort kann nicht aus einer Sprache in die andere entlehnt sein, und an besondere Berührungen zwischen Germanisch und Litu-Slavisch glaube ich nicht, s. o. S. 62. Der Lachs kommt bekanntlich nur in den Flüssen vor, die zu den nördlichen Meeren fließen. Das Wort mußte also den südlichen Sprachen verloren gehen. Ich würde lachs für ein idg. Wort halten, auch wenn es nicht im Slavisch-Lit. vorkäme, weil die Bildung aus germ. Sprachgut absolut unerklärbar ist. Das Wort muß aber schon die Entwicklung der Gutturale zu Zischlauten mitgemacht haben.

Wir besitzen ferner die Gleichung altpr. kalis, mhd. wels und Walfisch, die wir ebenfalls als indogerm. ansprechen dürfen, selbst wenn nicht lat. squalus, vgl. Walde Etym. Wtb. s. v., dazu gehört. Osthoff Etym. Parerga S. 321 hat in ausführlicher Begründung griech. $\varphi \alpha ́ \lambda \lambda \alpha \imath v \alpha \mathrm{zu}$ wal gestellt.

Lidén hat uns noch eine Reihe anderer Fischnamen kennen gelehrt. Btr. 15, 509 findet sich die Gleichung nnord. harr 'die Asche', lit. karšìs 'der Brachsen', kiršlỹs 'Äsche’. Uhlenbeck hat Arkiv f. nord. fil. 15, $154 \mathrm{f}$. nschw. gärs 'Kaulbarsch', nachdem Lidén es Btr. 15, 508 besprochen hatte, zu ai. jhașás gestellt, was er auch in seinem Et. Wtb. des Aind. beibehält. Torbiörnson die gemeinslavische Liquidametathese 35 hat dazu russ. żérech 'Seepferdchen' gefügt.

Das deutsche Stör, ahd. sturio stimmt in seinen Lauten auffällig zu abg. jesetrü russ. osëtrü, lit. asétras. Die indogerm. Grundform würde ich als oséteros ansetzen, aus der sich die slav. und germ. Formen durch Ablaut ergeben. Die Gleichung ist schon früher veröffentlicht worden, ich weiß aber nicht von wem.

In den Uppsalastudier S. 99 hat Lidén ferner aisl. $h \bar{a} r$ 'Haj' mit aind. çarkúş 'ein best. Wassertier' (unbelegt) verglichen. Dazu auch çakulás 'ein Fisch'. Auch Uhlenbeck hat diese Etymologie in seinem aind. Wtb. aufgenommen. Sie ist gewil nicht sicher, aber zweifellos möglich. Griech. kn̂toc kann wohl mit Lidén auch dazu gestellt werden.

Geben wir die Gleichung d. wal l. squälus auf, so findet doch dieses einen Verwandten in griech. ckúliov 'eine Haifischart', vgl. Osthoff Etym. Parerga 325.

Ich stelle ferner kelt. esox 'Lachs' zu deutsch Asche, Äsche. Daß der Fisch im Germ. nach seiner aschgrauen Farbe benannt 
wäre, ist mir wenig wahrscheinlich, da der Fisch gar nicht besonders grau ist. esoks und ahd. asko bilden eine tadellose Gleichung mit Schwebeablaut und müssen schon deshalb in die Ursprache zurückgehen. Ich bemerke noch, daß die Asche zu den Lachsfischen gerechnet wird.

Mit ahd. forhana 'Forelle' hat Lidén Uppsalastudier S. 92 ir. ork (aus *pork) 'salmo' und weiter lat. perca, griech. $\pi$ '́ $\rho$ kn 'Barsch' verglichen. Daß der Name im letzten Grunde mit griech. $\pi \epsilon \rho \kappa v o ́ c$ 'bunt', ai. prçni- 'gesprenkelt' zusammenhängt, ist möglich, aber es kann dieser Zusammenhang auch so aufgefaßt werden, daß *perknós von *perk 'Forelle' abgeleitet ist.

Schrader RL. 332 hat selbst einen Namen für den Hering entdeckt; die Gleichung ir. scatan, sgadan 'allec', nir. sgadan, manx. skeddan, kymr. ysgadan, ags. sceadd, engl. shad, norw. skadd, nhd. (mundartl.) schade, schaden kann sehr wohl urverwandt sein.

Mhd. smerl, smerle wird bei Kluge mit griech. cuapíc 'ein kleiner gering geachteter Meerfisch' verglichen, wogegen garnichts einzuwenden ist. Die Gleichung russ. sigü, an. sikr 'salmo lavaretus'steht bei Schrader RL. 495. Man sieht also, daß eine ganz beträchtliche Zahl von Fischnamen vorliegt, die ebensogut indogermanisch zu gelten ein Anrecht haben, wie andere Gleichungen.

Der einzige auffällige Punkt ist dabei, daß die Gleichungen sich nur in wenigen Sprachen erhalten haben. Aber man brancht zur Erklärung nur an die heutigen Verhältnisse zu denken. Wer nur einigermaßen gereist ist, weiß, wie die Fischnamen von Gegend zu Gegend wechseln. Derselbe Fisch heißt hier so, dort so. Außerdem sind gewisse Fische auf bestimmte Gegenden beschränkt. Für den Hering kann ein Ausdruck im Süden nicht vorhanden sein, für den Felchen keiner im Norden usw. Daraus läßt sich also nichts folgern.

Man kann nun auch einmal die ganze Frage von einer andern Seite betrachten. Man kann fragen, was besitzen wir in den Einzelsprachen an Fischnamen und woher stammen sie? Aus den modernen Dialekten ließe sich sicher ein großes Material zusammenbringen, ich kann aber darauf nicht eingehen. Ich wähle zunächst eine Reihe altdeutscher Fischnamen, die im Summarium Heinrici stehen (Steinmeyer-Sievers Ahd. Glossen 3, 83).

ipoccus : hüsc, nach Kluge auch ndd. bezeugt. Daß das Wort mit čech. poln. wyz zusammenhängt, ist klar, doch kann wohl nur das slavische Wort aus dem Germanischen entlehnt sein. 
Es ist bis jetzt keine Ableitung des germ. Wortes gelungen, und es sieht auch sehr altertümlich aus.

rombus : sturo, sturio, ndl. steur, ags. styrie, (styra), Etymon nach Kluge dunkel, siehe aber oben S. 69.

esox : lahs, idg. s. o. S. 69.

gamarus : salmo, nach Kluge aus dem Keltischen, aber dort nicht nachgewiesen; lat.-gall. salmo ist wahrscheinlich ein Lehnwort. Ich sehe eigentlich keinen Grund, das deutsche Wort für ein keltisches Lehnwort zu halten, da es gut deutsch aussieht. capito: ahd. alant oder munua. Das zweite Wort ist ganz unklar, das erste zu as. alund, an. olunn 'ein Fisch'. Ursprung dunkel. Über die Verwandtschaft mit dem Worte Aal s. o. S. 68. clama : ag ist mir unklar.

lucius : hechit, as. hacud, ags. hacod. Nach Kluge zu ahd. hecken 'stechen', also 'der Stecher'. Das ist möglich, aber das Suffix ist selten, und die Bildung sieht recht altertümlich aus. Es heißt auch, wie ich aus Brehm entnehme, schnöck, schnock, Wasserwolf.

porca : bersich, ndl. baars, ags. bcers, schwed. abborre, dän. aborre, nach Kluge zu borste, bürste gehörig. Die Ableitung ist wieder dunkel.

timallus : ascho, vgl. oben S. 69.

tactuca : forhana, s. 0.

anguilla: Aal, s. o.

ysmerenna, grece myrena. lanpreda, entlehnt.

gracius : chresse 'Grundling' unklar

turonilla : grundela zu grund

balene : walirun, cete : wal, s. o.

Charakteristisch ist, daß sich Entlehnungen unter diesen Fischnamen so gut wie gar nicht finden und nur wenige deutliche Ableitungen.

Außer den im ahd. belegten Worten gibt es aber noch eine ganze Reihe anderer.

Ahd. karpfo, ndl. karper, anord. karfe, spätlat. carpa, frz. carpe, ital. carpione, russ. korop, serb. karp, lit. kárpa. Das Wort ist im Germanischen wahrscheinlich ein Lehnwort, worauf schon das inlautende $p$ hinweist. Es hindert aber nichts frz. carpe, und russ. kóropŭ zu vereinigen. Uhlenbeck hat $\mathrm{PBrB} .19,331$ mit unserm Wort ai. çaphara-, caphari, ein häufig belegtes Wort für eine Karpfenart verglichen, für das er Dissimilation aus 
*garphara annimmt. Dagegen ist garnichts einzuwenden. Schrader sagt zwar RL. 409, die Anknüpfung habe wenig Wahrscheinlichkeit, aber, wenn es sich nicht um einen Fischnamen handelte, würde er die Gleichung sehr wohl billigen.

Ahd. slio, ags. sliw 'Schleie' nach Kluge zu Schleim wegen der schleimigen Schuppen, was mir kaum glaublich ist. Eher ist der Fisch nach der Farbe benannt. 'Gewöhnlich' sagt Brehm, zeigt das Kleid der Schleihe ein dunkles Ölgrün, durch welches ein schimmernder Goldglanz geht. Daher könnte man germ. *slīwas zu lat. livveo, livor, abg. slivva 'Pflaume' stellen. Aber das Wort hat auch Verwandte im Lit.-slav. nämlich lit. lýnas, altpr. linis, le. lins, abg. lini. Berneker Die preußische Sprache S. 304 stellt

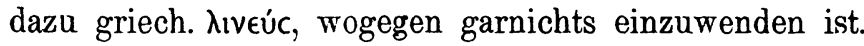

Weiter liegt ein wgerm. Fischname vor in ndd.ruche, ndl. roch, ags. reohha. Auch dieses Wort hat ein höchst altertümliches Aussehen. Ein Zusammenhang mit lat. rāja ist allerdings kaum möglich.

Außerdem wäre noch der Butt zu nennen. Auch hier ist eine etymologische Anknüpfung noch nicht gefunden.

Das Elbinger Vokabular bietet uns ebenfalls eine Fülle von Fischnamen. Es ist vielleicht angebracht, auch diese hier anzuführen, damit man erkennt, wie sich einheimisches Gut zum fremden verhält. Erwähnt sind schon suckis 'Vysch' zu lit. żuvis, lasasso 'Lachs', angurgis 'ael', esketres 'Stör', kalis 'Wels', linis 'slye'. Die andern sind: liede 'Hecht', lit. lydekà, le. lìdaka, lìdeks.

locutis 'Brassen'.

starkis 'Zant', lit. stérkas, le. starks.

wilnis 'Quappe': das von Berneker verglichene litt. wilnis 'Pilz' gehört kaum dazu.

smerlingis 'schmerle' aus dem Deutschen.

seabre 'Czerte', d. i. 'Zärte', lit. žobrỹs, lett. zïbris.

assegis 'Persk', d.i. Barsch, lit. ežeg ỹs 'Kaulbarsch', poln.jaždž.

brunse 'Pletze', lit. brunszis; sylecke 'Hering', lit. sileke, lett. silkis; sarote 'Karpfen', lit. žarotas 'schimmernd'; blingis 'Blei', blingo 'Mutterlosen'; grundalis 'Grundel' entlehnt; malkis 'Stint'; dubelis 'Halbfisch' aus deutsch döbel; stroysles 'Tobel'; rapis 'Rape'; sweikis 'Dorsch'.

Auch hier ist das meiste unklar, einige Fischnamen sind entlehnt, aber das meiste macht doch einen recht altertümlichen Eindruck. Vielleicht gelingt es mit der Zeit noch einen oder den anderen aufzuklären. 
Bekanntlich handelt fast das ganze siebente und achte Buch des Athenaios von den Fischen. Die Fülle der dort aufgezählten Namen ist zu groß, als daß ich sie hier anführen könnte. Es finden sich darunter viele ganz verständliche Bildungen, andere Worte machen aber einen höchst altertümlichen Ausdruck.

Aus andern Sprachen fehlen mir Sammlungen.

Um nun schließlich das Maß vollzumachen, besetzen wir im Germ. ein Wort für den Fischrogen ahd. rogan, an. hrogn, engl. roan. Das Wort ist, ich weiß nicht von wem, mit lit. kurkula $\tilde{\imath}$ 'Froschlaich' verglichen. Die Vergleichung ist jedenfalls tadellos.

"Auf keinen Fall können die Indogermanen ausschließliche Fischesser gewesen sein ... oder auch nur dem Fischfang oder dem Fischgenuß eine besondere Aufmerksamkeit zugewendet haben". Warum denn nicht? Aus der Sprache können wir weder dies noch das Gegenteil beweisen. Waren denn aber die Fische ein Nahrungsmittel, das überall zur Verfügung stand? Nein, sondern es gab sie nur an gewissen Stellen in bedeutender Menge, und da werden die Menschen sie auch schon benutzt haben. Wir wissen dies von den Bewohnern der dänischen Küchenabfälle, und auch Bewohner der friesischen Inseln, sowie der Nordseeküste werden sie nicht verschmäht haben. Ebenso hat es bei den Griechen fischessende Bevölkerungsschichten gegeben, soweit sie eben an dafür geeigneten Stellen saßen.

Aus dem vorhergehenden folgt also mit Sicherheit, daß es indogermanische Fischnamen gibt, und daher fällt das ganze Gebäude Schraderscher Schlußfolgerungen zusammen. Die prinzipielle Frage ist daher hier noch nicht zu entscheiden.

\section{B. Schiffahrt und Wagenbau.}

Es ist bekannt, daß wir eine ausgebildete Terminologie für den Wagen haben. Der reichen Fülle gegenüber soll die Armut in bezug auf die Terminologie der Schiffahrt ins Gewicht fallen. Nun steht doch über allem Zweifel fest, daß die Indogermanen Worte für Schiff und Ruder besessen haben, aber das genügt Schrader nicht. RL. S. 711 sagt er: "Wo immer ein Volk, wenn auch neben andern Beschäftigungen, Jahrhunderte lang dem Gewerbe der Schiffahrt obliegt, wird sich unfehlbar auch eine nautische Terminologie herausbilden. Für die charakteristischen Merkmale der Seelandschaft, für das Wetter auf See, für die bedeutendsten Seetiere, für die Winde, für die 
Himmelsgegenden, für den Fischfang, für Arten und Teile der Fahrzeuge usw. werden feste Namen geschaffen werden, wie dies uns handgreiflich in dem urgermanischen Sprachschatz entgegentreten wird. Wären derartige Wörter nur in einigem Umfang schon in der idg. Grundsprache vorhanden gewesen, so würden, wie auf dem Gebiete der Viehzucht und des Ackerbaus, die Spuren derselben in idg. Gleichungen vorliegen. Solche fehlen aber, von den obigen abgesehen, nahezu gänzlich".

Vielleicht werden sich einige durch diese schön gesetzten Worte bestechen lassen; wer aber wirklich einmal das Meer und die Schiffahrt gesehen hat, dem wird das Haltlose dieser Ausführungen sofort klar werden. Ist denn Ackerbau und Viehzucht mit der Schiffahrt auf eine Linie zu stellen? Nein, denn Ackerbau und Viehzucht sind die Grundbedingungen aller Wirtschaft durch fast ganz Europa hindurch, die Schiffahrt ist aber nur an wenigen Stellen möglich, nämlich am Meer und auch hier nur, wo Häfen vorhanden sind, auf Seen und Flüssen. Es hat also immer nur ein kleiner Teil der Indogermanen die Schiffahrt betreiben können, die Hauptmasse, die im Binnenland saß, aber nicht. Tatsächlich mußten die Völker, die von der See ins Binnenland zogen, ihre nautischen Fertigkeiten und ihre nautischen Ausdrücke aufgeben, darüber sind wir doch alle einig. Man vergleiche z. B., daß got. saiws 'Landsee' heißt gegenüber der Bedeutung 'Meer' in den übrigen Sprachen. Wir wissen ferner, daß die gemeingermanischen Ausdrücke, die sich auf die See beziehen, im Oberdeutschen vielfach fehlen. Selbst wenn wir nicht einmal ein idg. Wort für Schiff erschließen könnten, würde daraus folgen, daß die Bewohner der Nord- und Ostseeküste keine Indogermanen gewesen wären? Auch der Vergleich mit der Terminologie des Wagens hinkt, weil eben der Wagen in der Landwirtschaft immer gebraucht wird, das Schiff aber nicht die gleiche Verbreitung hat.

Außerdem ist die nautische Terminologie keineswegs so arm, wie das Schrader hinstellt.

Außer dem idg. Wort näus haben wir noch griech. raulóc, ahd. kiol 'großes Schiff', altn. kjöll, ags. cēol.

Wir haben nicht nur einen Ausdruck für 'Ruder', sondern wahrscheinlich zwei, neben ée€tuóc steht an. ags. ār, das Lidén Studien zur aind. und vergl. Sprachg. S. $65 \mathrm{zu}$ lit. waĩras, waĩra, lett. airis gestellt hat. 
Deutsch mast entspricht lat. mālus. Schrader meint nun zwar RL. S. 755, man könne hier von der Bedeutung 'Stange' ausgehen, die im ir. maide $={ }^{*}$ masdos 'lignum, baculus' die einzig herrschende ist. Ich muß es für den größten Zufall erklären, wenn zwei Worte in ihrer Bedeutungsentwicklung derartig sollten zusammengetroffen sein, vgl. meine Indogermanen S.239. Dagegen ist es ganz leicht verständlich, wenn ein Ausdruck für Mast in die Bedeutung 'Stange' umschlägt. Wir reden ja auch von Fahnenmasten usw. Schrader sagt nun zwar, im Lateinischen und im Althochdeutschen sei die Bedeutung 'Stange, Baum' noch so lebendig, daß nichts im Wege stehe, diese als die ursprüngliche anzunehmen.

Die Tatsachen liegen folgendermaßen. Für das Ahd. belegt Graff mast malus Tr, malum VA. V.487, mastin malis AId. 1, mastboum. Erst im Mhd. tritt die Bedeutung 'aufrecht stehende Stange' auf, wie das DWB. angibt. In der ags. Poesie ist moest in der Bedeutung 'Mast' überliefert B. 1898, Gn. C. 24, B. 36, 1905, An. 465, und nur an einer Stelle Gen. 1470 bedeutet es Baumstamm, Ast. Hier ist aber die Überlieferung nicht in Ordnung. Es heißt dort bei Grein

\section{gefeah blidemod,}

poes pe heō gesette swïte wërig on treowes telgum torhtum moste.

Man will hier moste für moste lesen, besser ist aber wohl mit Grein moste als Verbum zu nehmen, und gesette in gesittan zu ändern, wie Wülcker auch tut. most für moest wäre doch sehr auffallend.

Wenn im Nordischen mastr in älterer Zeit nicht vorhanden ist, sondern dafür siglutrē gebraucht wird, so wird doch damit. nicht bewiesen, daß das Wort nie vorhanden war, sondern es ist einfach, wie das so oft geschieht, durch eine Umschreibung ersetzt worden.

Es ist bedauerlich, daß für das Lat. malus der thesaurus. noch nicht vorliegt. Aber schon aus Georges ist zu ersehen, daß Schrader kaum recht hat. Georges gliedert die Bedeutungen so: mälus 'jeder senkrecht stehende Balken, ein Ständer' I im allgemeinen, und das wird mit einer Stelle aus Frontin. strat. 3, 8, 3 belegt: malos exaequantes altitudinem jugi surrexit, II insbes. der Mast, Mastbaum. Daß diese hier angenommene Bedeutungsentwicklung höchst unsicher ist, muß sich jeder sagen. 
Ich habe nun noch den Forceilini nachgeschlagen und dort keinen einzigen Fall gefunden, der für Schrader spräche, sondern es liegt überall die Bedeutung 'Mast' vor.

Man sieht also, was es mit der Behauptung Schraders auf sich hat, im Althochdeutschen und Lateinischen sei die Bedeutung 'Stange, Baum' noch so lebendig, daß nichts im Wege steht, diese für die ursprüngliche anzunehmen.

Es ist also auch für das Lateinische höchst wahrscheinlich von der Bedeutung 'Mast' auszugehen, und da diese auch für das Germanische zugrunde liegt, so rechtfertigt die Sprache die Annahme, daß die Indogermanen den Mast gekannt haben. $\mathrm{Ob}$ die Sprache gegenüber andern Indizien Recht behält, ist eine andere Frage, die ich in meinen Indogermanen behandelt habe.

Wenn nun die Indogermanen 'Schiffe' mit 'Rudern' und 'Masten' besessen haben, was haben sie damit gemacht? Sie haben die Flüsse befahren, werden auch die See nicht gescheut haben, wenn sie an ihr saßen.

Schrader weist mit Emphase auf die Fülle urgermanischer Worte hin, die sich auf die Seefahrt beziehen, und die die Bekanntschaft der Germanen mit der Seeschiffahrt erweisen. Er hätte aber die indogermanischen Ausdrücke nicht übergehen sollen.

Ich stelle diese daher zusammen

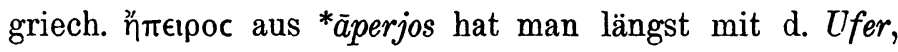
mhd. wover, mndd. over, ndl. oever, ags. ofer verglichen. Die Gleichung ist tadellos, und es kann demgegenüber die oberd. (baier.) Form urvar garnicht in Betracht kommen. Da das Wort den oberdeutschen Dialekten noch heute fremd ist, man sagt dafür 'Staden', so wird es sich bei urvar um eine Volksetymologie handeln.

Lat. portus 'Hafen' entspricht ganz genau aisl. fjordr 'Bucht'. In andern Dialekten liegt die Bedeutung 'Furt' vor, aw. pošus 'Furt', porotuš 'Durchgang, Eingang, Pforte, Furt, Brücke', ahd. furt, gall. ritu-

ai. ürmiş̧ 'die Welle, Woge', entspricht ags. wȳlm m. flodes, wateres wylm

d. welle, ahd. wëlla kehrt wieder in lit. vilnis, abg. vlüna 'Welle' ags. lagu, lat. lacus, air. loch bilden eine tadellose Entsprechung. Das Wort bezieht sich offenbar auf einen Landsee.

Namen für die Himmelsgegenden sind nicht bloß germanisch, sondern schon indogermanisch. Es entspricht bekanntlich lit. 
šiaurỹs 'Nordwind', abg. sèverü 'Nord', lat. caurus 'Nordostwind' dem ahd. skūr 'Ungewitter', nhd. schauer, got. skūra windis 'W irbelwind', ags. skūr 'Schauer'. Die besondere Entwicklung des Germanischen ist leicht verständlich, da der Nord- oder Nordwestwind meist zu böigem oder stürmischem Wind ausartet. Für die Bewohner der Nordseeinseln sind die Nordwestwinde am gefährlichsten.

An der Gleichung d. süden, ahd. sundwint, ags. sütan 'von Süden her', ags. sūp, ndl. zuid, as. sūth 'Süden' mit griech. vótoc 'Südwind' aus *snotos halte ich durchaus fest.

Daß in zwei Fällen die Worte für Windrichtungen zur Bezeichnung der Himmelsgegenden geworden sind, halte ich für recht auffallend.

Auch sonst haben wir noch Wetterbezeichnungen: lat ventus, got. winds usw.; as. wëdar 'Wetter, Witterung, Sturm' entweder zu abg. vedro 'gutes Wetter' oder zu vétrú 'Luft, Wind'. Auch d. sturm kann ein altes Wort sein, da es im Germanischen schwerlich abgeleitet sein kann. Natürlich beziehen sich diese Worte nicht notwendig auf die Seelandschaft.

Schrader sagt weiter S. 715: Urgermanische Tiernamen der nördlichen Fauna s. u. Möwe, Schwan, Seehund, Walfisch. Für Walfisch läßt sich, wie wir oben gesehen haben, ein idg. Wort erschließen. Für den 'Seehund' bestehen eine Reihe dunkler Ausdrücke, griech. púkn, altn. selr, ags. seolh, ahd. selah. Letzteres hat man zu griech. célaxoc 'Knorpelfisch' gestellt. Das ist aus bekannten Gründen nicht ganz sicher. Immerhin sieht aber germ. selh recht altertümlich aus.

Für 'Schwan' haben wir in ahd. albiz, abg. lebedi eine sichere idg. Gleichung, weil sie Ablaut zeigt.

Von den verschiedenen Bezeichnungen für 'Möwe' sieht die germ. ahd. $m e \bar{h}$, altn. $m \bar{a} r$, ags. $m \bar{e} w$ recht altertümlich aus, wenngleich sich bei der beschränkten Verbreitung dieser Tiere naturgemäß kein weit verbreiteter Name nachweisen läßt.

Die größere Bedeutung des Fischfangs macht sich geltend, sagt Schrader, in urgermanischen Gleichungen wie altn. ongull, abd. angul 'Angel', got. nati, ahd. nezzi 'Netz', altn. vadr, mhd. wate 'Zugnetz', altn. hrogn, ahd. rogan 'Rogen', dän. leeg, mndd. $l e \bar{k}$ ' 'Laich'.

Von diesen Worten entspricht angul dem griech. árkúloc 'gekrümmt, gebogen' ganz genau und von dem zugrunde liegen- 
den Stamm ist tò ărkıctpov 'Angelhaken' abgeleitet. Unser deutsches Wort netz hat wahrscheinlich in lat. nassa eine Verwandte. Wate kann ein neues germanisches Wort sein. Über Rogen s. 0. Über Laich weiß ich nichts zu sagen.

Die urgermanischen Fischnamen Aal, Lachs, Stör haben wir oben für indogermanisch erklärt. Für den Namen des Herings hat Schrader selbst den Anfang dazu gemacht, und so bleibt einzig der Barsch. sich hat.

Man sieht also, was es mit Schraders Ausführungen auf

Ich füge noch hinzu, daß eine Reihe anderer Seeausdrücke durchaus altertümlich aussehen, so ndd. düne zu ags. dün 'Hügel' (engl. downs 'Dünen'), wozu auch engl. down 'herab' aus ags. adūne ofdüne eig. 'vom Hügel herab'. Kluge vergleicht dies weiter mit air. dūn 'Hügel'. Dies Wort ist dann von den Germ. entlehnt als tūn, d. Zaun. Vielleicht ist aber unser 'düne' ver-

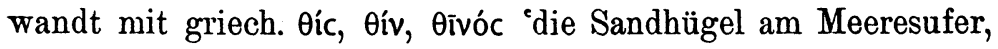
die Dünen'.

Das ndd. wat entspricht lat. vadum 'Furt'. Unser deutsches Wort Strand sieht höchst altertümlich aus, ebenso wie Ebbe.

Es gibt also eine Fülle für die idg. Ursprache zu erschließender Ausdrücke, die sich auf die Schiffahrt und die See beziehen, und diese machen es durchaus wahrscheinlich, daß die Indogermanen an der See gewohnt haben. Jedenfalls sind aber auch hier wieder die Schlüsse aus dem Schweigen der Sprache hinfällig.

\section{Blumenzucht und Ackerbau.}

Daß die Indogermanen keine Blumenzucht getrieben haben, folgt natürlich nicht aus dem Mangel an Ausdrücken dafür wir haben ja Ausdrücke für Blume -, sondern aus andern kulturhistorischen Momenten. Ich denke, ich kann diesen Punkt ganz übergehen.

D. Die Verschwägerungsbezeichnungen des Mannes

$$
\text { gegenüber denen des Weibes. }
$$

Delbrück und Schrader haben ziemlich gleichzeitig den Nachweis aus der Sprache zu führen versucht, daß die Verschwägerungsbezeichnungen sich auf das Verhältnis der Frau zu den Angehörigen des Mannes beziehen, und daß wir daher ein rein agnatisches Verhältnis für die Urzeit anzunehmen haben. 
Diese Ausführungen haben ursprünglich auch auf mich Eindruck gemacht, und ich habe ihnen einige Beweiskraft beigemessen. Heute muß ich das zurücknehmen, und ich will zu zeigen versuchen, daß Schraders Folgerungen - die Delbrücks gehen ja nicht so weit - falsch sind.

Es ist sicher, daß die Verwandtschaftsbezeichnungen im Laufe der Zeit immer mehr verringert worden sind, weil man kein Bedürfnis hatte, die einzelnen Grade noch so stark zu unterscheiden, wie man in alten Zeiten tat. Heute kommen wir mit sehr wenig Worten aus, weil sich unsere Familienformen gelockert haben.

Wollen wir nun ermitteln, welche Bezeichnungen in alter Zeit nötig waren, so tut man zunächst gut, sich einmal an die Völker zu wenden, die die alten Verwandtschaftsnamen und die alten Formen der Familie am besten erhalten haben, das sind die Litauer und die Slaven. Bei den heutigen Südslaven besteht noch die Großfamilie, und sie besitzen die alten Ausdrücke djëvêr, zaova, jëtrve u. a. Aber sie haben eine ausgebildete Nomenklatur der Verwandtschaftsworte nicht etwa nach einer Seite, sondern nach beiden. Es ist wichtig, das einmal übersichtlich zu zeigen, vgl. Delbrück S. 404.

$\begin{array}{lll}\text { Vater } & \begin{array}{c}\text { Litauer } \\ \text { té̀ras }\end{array} & \begin{array}{c}\text { Slaven (Serben) } \\ \text { otac }\end{array} \\ \text { Mutter } & \text { motina } & \text { mati } \\ \text { Sohn } & \text { sūnùs } & \text { sin } \\ \text { Tochter } & \text { duktẽ } & \text { hci } \\ \text { Bruder } & \text { brolis } & \text { brat } \\ \text { Schwester } & \text { sesũ } & \text { sestra } \\ \text { Ältere Schwester } & & \text { ljelna } \\ \text { Vaters Bruder } & \text { dedis } & \text { stric } \\ \text { dessen Frau } & \text { dedéne } & \text { strina } \\ \text { des Vaters Schwester } & \text { dede } & \text { strina, tetka } \\ \text { ihr Mann } & \text { dedens } & \\ \text { der Mutter Bruder } & \text { avynas } & \text { ujak } \\ \text { seine Fran } & \text { avynene } & \text { ujna, ujača } \\ \text { der Mutter Schwester } & \text { tetta } & \text { teta, tetka } \\ \text { ihr Mann } & \text { tettenas } & \text { tetak, tetac } \\ \text { des Weibes Vater } & \text { uożvis } & \text { tast, punac } \\ \text { des Weibes Mutter } & \text { uożvé } & \text { tasta, punica } \\ \text { des Mannes Vater } & \text { šéšuras } & \text { svekar }\end{array}$


H. Hirt,

$\begin{array}{lll}\text { des Mannes Mutter } & \text { anýta } & \text { svekrva } \\ \text { des Mannes Bruder } & \text { deveris } & \text { djever } \\ \text { des Mannes Schwester } & \text { mõzza } & \text { zaova } \\ \text { des Weibes Bruder } & \text { laigonas } & \text { šura } \\ \text { des Weibes Schwester } & \text { svaine } & \text { svast } \\ \text { Männer zweier Schwestern } & \text { svainis } & \text { pašenog } \\ \text { Frauen zweier Brüder } & \text { gente } & \text { jetrve } \\ \text { Eidam } & \text { žentas } & \text { zet } \\ \text { Schnur } & \text { marti } & \text { snaha. }\end{array}$

Man könnte ja einmal versuchen, ohne Rücksicht auf die übrigen Sprachen den urlitauisch-slavischen Stand der Dinge zu erschließen. Es fehlten dann Ausdrücke für Vater, für den Bruder und die Schwester des Vaters, für die Schwiegertochter; sie wären aber vorbanden für die Mutter, den Bruder und die Schwester der Mutter, und für den Schwiegersohn. Wenn das nicht auf Mutterrecht weist; so weiß ich nicht, was es anders bedeuten soll. In Wirklichkeit ist natürlich die Schlußfolgerung falsch.

Die Fülle der Verwandtschaftsbezeichnungen setzt einigermaßen in Erstaunen, aber da schließlich die Litauer und Slaven nicht die Indogermanen sind, so haben diese wahrscheinlich noch mehr besessen. Jedenfalls liegt kein Grund vor, anzunehmen, erst die Litauer und Slaven hätten das Bedürfnis empfunden, derartige ausgeprägte Verwandtschaftsbezeichnungen zu schaffen. Es ist sehr wohl möglich, daß alle diese rerschiedenen Unterscheidungen im Indogerm. vorhanden waren, daß aber in den Einzelsprachen eine Reihe davon verloren ging, weil eine immer größere Verallgemeinerung eintrat. So waren *swek'uros und swek'vā ursprünglich die Bezeichnungen für den Schwiegervater und die Schwiegermutter der Frau, sie wurden aber auch allmählich für das Verhältnis des Schwiegersohnes zu seinen Schwiegereltern gebraucht, unter Verdrängung anderer Ausdrücke. Tatsächlich bezeichnen alle Sprachen dies Verhältnis irgend wie, und da die Sachrergleichung das wichtigere ist, so ist darans $\mathrm{zu}$ schließen, daß es auch im Indogermanischen bezeichnet wurde. Wir sind nur nicht imstande, die indogermanischen Ausdrücke festzulegen, weil die Sprachen auseinandergehen.

Sehen wir uns nun die sprachlichen Tatsachen noch etwas näher an. 
Der Ausdruck für den Bruder des Vaters ist nur in

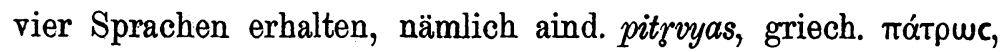
1. patruus, ahd. fetiro.

Für den Mutterbruder ist zwar ein so genau übereinstimmender Ausdruck nicht vorhanden. Aber fünf Sprachen bezeichnen ihn mit demselben Stamm awo, lat. avunculus, kelt. eviter, germ. ōheim, lit. avynas, slav. uji. Daß das kein Zufall sein kann, dürfte mit Delbrück für jeden außer Schrader auf der Hand liegen. Es wäre höchst merkwürdig, wenn alle Völker denselben Stamm gewählt hätten.

Daß für die Schwiegertochter ein alter Ausdruck besteht, ist sicher, aber ebenso so sicher auch für den Schwiegersohn. Die Übereinstimmung zwischen ai. jāmātar, av. zāmātar, griech.

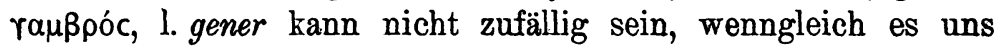

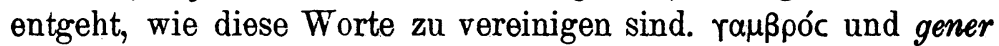
würde man unter Annahme eines idg. ${ }^{*} g^{e} m n r o s$ vergleichen können. Ebenso decken sich ai. jāmātar, falls aus jāmit mit späterem Suffix -ar mit lit. slav. ${ }^{*}$ żenət, falls man $\hat{g}^{e} m n ə t$. als Grundform annimmt. Aber wie dem auch sein mag, es ist mir ganz unglaublich, daß alle Sprachen selbständig auf denselben Stamm zur Bildung dieses Namens verfallen sein sollten.

Man darf doch nicht vergessen, daß sich auch bei uns im Lauf der Zeiten die Ausdrücke geändert haben. Das alte Wort 'Eidam' ist verschwunden und durch 'Schwiegersohn', 'Tochtermaun', engl. son in law ersetzt. Da englisch und deutsch auseinander gehen, so müßte man wieder schließen, daß die Germanen kein Wort für 'Schwiegersohn' gehabt hätten.

Für die Schwiegereltern des Mannes sollen sich keine Ausdrücke nachweisen lassen. Nur wir finden ai. çvácuras, gvaçrí, 1. socer, socrus, alb. vịehar, viéhar̄o, germ. Schwäher und Schwieger, d. h. in vier Sprachen dient ein und dasselbe Wort auch zur Bezeichnung der Schwiegereltern des Mannes. Es kann daher auch so schon im Indogermanischen gewesen sein. Wir finden außerdem griech. $\pi \epsilon v \theta \epsilon \rho o ́ c, \pi \epsilon v \theta \epsilon \rho \alpha ́$, lit. üšvis, üšvé und abug. tistĭ, tista. Wenn nun auch diese Worte nicht übereinstimmen, so kann doch eines und das andere schon im Indogermanischen in dem Sinne von Schwiegervater gebraucht worden sein. Wenn ich aber wie Schrader schließen wollte, so würde ich sagen; im Indogermanischen bezeichnete ékupóc auch den Vater der Frau, und erst in einigen Einzelsprachen ist das Bedürfnis aufge-

Indogermanische Forschungen XXII. 
kommen, den Schwiegervater der Frau durch ein besonderes Wort zu bezeichnen. Das ist in einzelnen Fällen durchaus möglich. Man sieht also, wie wenig Schraders Schlußfolgerungen wirklich das beweisen, was sie beweisen sollen.

Wir müssen aber noch auf einen anderen Punkt eingehen. IF. 17, 11 hat Schrader über Bezeichnungen der Heiratsverwandtschaft bei den idg. Völkern gehandelt. Dieser Aufsatz bedarf dringend einer eingehenden Besprechung, da er eine ganze Reihe höchst zweifelhafter und evident falscher Dinge enthält. Die Ausführungen über das deutsche Wort schwāger bilden freilich den Gipfelpunkt der Schraderschen Methode. Dieses Wort soll aus dem Slavischen swāk entlehnt sein. Dann sei es nach $m \bar{a} c$, māges $\mathrm{zu}{ }^{*} s w a \bar{k}, *^{*}$ swāges und schließlich unter Einfluß von swiger und sweher zu swäger umgestaltet. Zunächst ist es schon höchst zweifelhaft, ob die Germanen solch Wort von den Slaven haben entlehnen können, und der Zweifel wird in keiner Weise dadurch beseitigt, daß Schrader ein paar Entlehnungen des Germanischen aus dem Slavischen für kulturhistorische Begriffe anführt, nämlich für Pelze und Pelztiere und einige Namen für Vögel und Fische. Glücklicherweise istSchraders Annahme durch W. Schulzes Ausführungen KZ. 40, 400 definitiv beseitigt worden, und es lohnt sich nicht, weiter darauf einzugehen. Schwäger enthüllt sich als eine indogermanische Bildung gleich ai. gvāçuras ${ }^{\circ}$ dem Schweher gehörig'.

Schrader nimmt weiter an, daß es einen Ausdruck für

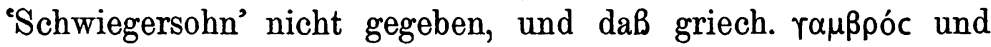
ähnliche Worte zunächst 'Heiratsverwandter' bezeichnet habe. Das ist deshalb durchaus unwahrscheinlich, weil wir bei den Verwandtschaftsnamen durchaus das Prinzip der Verallgemeinerung finden. Die beiderseitigen Schwiegereltern werden schließlich mit einem Ausdruck bezeichnet, ebenso die Oheime und

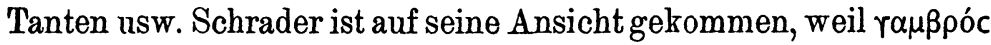
'Schwiegersohn, Schwager' und schließlich auch 'Schwiegervater' bedeutet. Daß die Bedeutung 'Schwiegersohn' in die von 'Schwager' übergehen kann, ist außerordentlich leicht verständlich. Wie leicht kann ein erwachsener Sohn von unserm 'Schwiegersohn' reden. Daß dieser Punkt gar nichts beweist, zeigt auch vuóc, das gleichfalls in dem doppelten Sinne der Schwiegertochter und der Schwägerin vorkommt. Hier wissen wir, daß die erste Bedeutung die alte ist. 


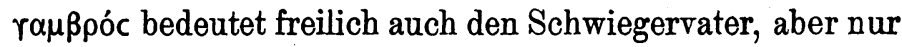
in poetischen Texten (Delbrück S. 115). Delbrück erklärt dies durch Anredewechsel, und das genügt vollständig trotz des Widerspruches von Schrader. Es kommt hinzu, daß ahd. suehur auch im Sinne von levir belegt ist. Voraussetzung für diese Bedeutungsübergänge ist nur, daß die ursprüngliche Bedeutung verblaßt ist.

Es ist also eine durchaus gewöhnliche Bedeutungsentwicklung, die wir vor uns haben, und jeder, der die Belege voreingenommen prüft, wird mit Delbrück zu der Ansicht kommen, daß eben eine einfache Bedeutungserweitung rorliegt, wenn wir die Ausdrücke für 'Schwiegersohn' auch in der Bedeutung 'Schwager' finden.

Natürlich können immer wieder neue Worte für den Schwager oder den Schwiegersohn aufkommen, auch Worte, die einst eine allgemeine Bedeutung gehabt haben.

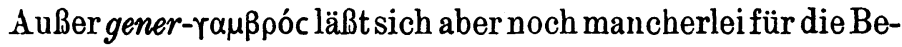
zeichnung derVerwandtschaft nach der weiblichen Seite anführen.

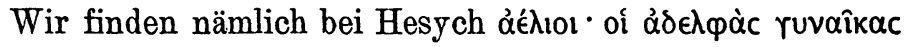

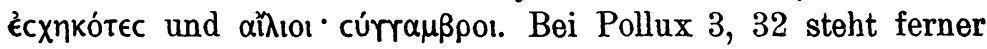

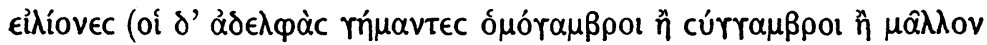

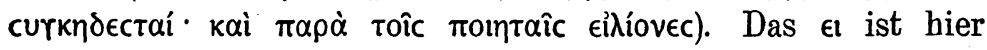
allerdings unklar, aber da es ein dichterischer Ausdruck ist,

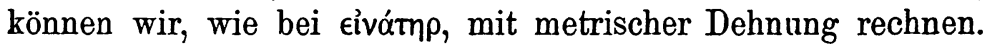
Wir kommen also zu é̉íovec. Die Wörter áélıo usw. dürften das bekannte Präfix $\alpha$ enthalten. Mit diesem Wort hat nun schon Kluge KZ. 26, 86 an. svili 'a brother in law', Pl. svilar 'the husbands of two sisters' zusammengebracht. Wir finden im Indischen ferner syālá- 'der Frau Bruder'. Delbrück meint zwar, das Wort könne nichts mit den beiden erwähnten zu tun haben, aber so unbedingt ausgeschlossen scheint mir das nicht zu sein, da man den verschiedenen Anlaut vielleicht unter einem Ansatz swjvereinigen könnte. Jedenfalls folgt schon aus dem griechischen und germ. Wort, daß es eine Bezeichnung für die Männer zweier Schwestern gab. Allerdings kann sich eine solche auch in der Hausgemeinschaft des Mannes einstellen, wenn zwei Brüder oder Vettern zwei Schwestern geheiratet hatten, aber sicher ist das keineswegs. Es ist vielmehr wahrscheinlicher, daß diese Bezeichnung von der weiblichen Seite ausgeht. Delbrück hält es für durchaus möglich, daß ein Mann in die Familie der Frau hineintrat, und ich schließe mich dem durchaus an. 
Schließlich ist doch der Vorgang dem der Adoption zu vergleichen. Auch hier tritt ein Mann in eine fremde Familie.

Mit mhd. geswige, geswie, ahd. gesuio levir, sororis maritus u. a.' weiß Delbrück nichts anzufangen. Auch Schraders Deutung IF. 17,25 ist nichts weniger wie sicher. Ich wundere mich eigentlich, daß man die fast genaue Übereinstimmung mit lit. svainnis nicht erkannt hat. svainnis heißt im Lit. der Bruder meiner Frau, also genau das, was geswio auch in den meisten Fällen bedeutet, vgl. z. B. Meier Helmbrecht 1664 . svainis geht zwar nach der jo-Deklination, aber es ist sebr wohl möglich, daß hiex erst ein Metaplasmus vom Akk. Sing. stattgefunden hat, genau wie bei sênis 'der Alte', lat. senem, élnis 'Hirsch', slav. jelens u. a. Dann mußte also der Nom. ursprünglich *swaiō lauten, und das wäre eine einfache ablautende Form $\mathrm{zu}$ ahd. ge-swio. Die Annahme, daß swainis aus dem Slav. entlehnt (Leskien Nom. Bild. S. 371) scheint mir durchaus nicht erwiesen zu sein.

Um den Aufsatz von Schrader ganz zu erledigen, füge ich hier noch einen Punkt hinzu. Schrader will sich für seine Erklärung von swāger noch auf einer andern Entlehnung aus dem Slavischen stützen, ahd. eninchilī sei aus slav. vünukü entlehnt. Hier erheben sich schon lautlich einige Schwierigkeiten. Die slav. Grundform ist ononk- und daraus läßt sich enin trotz des Verweises auf poln. wnęk nicht herleiten. Wir müssen also bei der Herleitung aus dem Deutschen bleiben. Nun hat A. Zimmermann IF. 15, 339 ein paar schöne Belege beigebracht über das Verhältnis des Namens 'Großvater' zu 'Enkel'. Wir finden lat. aviaticus, das deutlich $\mathrm{zu}$ avos gehört; und auch ir. aue könnte auf *avios zurückgehen. Das ist alles ganz richtig, und nur in der Ansetzung der Bedeutungsentwicklung hat man geirrt. Was heißt denn aviaticus? Nun nach Beispielen wie vineaticus 'zum Weinberg gehörig', cēnaticus 'zur Mahlzeit gehörig' heißt es 'zum Großvater gehörig', 'Großvaters Kind' sozusagen. Was damit gemeint ist, braucht nicht erörtert zu werden. Genau dasselbe müssen wir für kelt. *avios annehmen, auch das bedeutet 'zum Großvater gehörig'. Unser deutsches enenkel wird also ebenso zu erklären sein. Wie ist hier die Suffixbildung aufzufassen? Kluge Et. Wtb. s. v. Enkel sieht in ahd. eninchili ein selbständiges Suffix wie in huoninkllin 'Hühnchen', lēuinklīn 'kleiner Löwe' u. a. Wilmanns Deutsche Gr. 2, 322 zerlegt sogar en-in-klîn, zu ano. Dann kämen wir aber immer nur auf die Bedeutung 'Großväterchen'. 
Aber dieses Suffix -inkilī muß doch irgendwie durch $\mathrm{Zu}$ sammensetzung entstanden sein, und da wir auch mhd. enenkel finden, so kann man wohl darin die ursprüngliche Form sehen, der ein noch ursprünglicheres anénkos zugrunde liegt. Auf diese Form weist ja auch slav. vünukü. Daß diese Worte zusammengehören, scheint mir klar zu sein. Da aber die Suffixe nicht stimmen, so wird man am ehesten an Entlehnung denken dürfen, nicht aber des Germanischen aus dem Slavischen, sondern umgekehrt, des Slavischen aus dem Germanischen. Die Suffixgestalt -ko ist im Germanischen zwar nicht häufig, aber doch genügend belegt, und zwar auch in der Bedeutung 'gehörig zu' so brwochach 'zona, balteum' zum bruoh gehörig, funko zu got. fön 'zum Feuer gebörig' u. a. So deute ich denn *aninko als 'zum Ahn gehörig'. Man kommt also ganz gut mit dem Deutschen aus, und diese Stütze der Schraderschen Ansicht ist hinfällig ${ }^{1}$ ).

Im Slavischen, wo wir am ehesten alte Verwandtschaftsausdrücke zu finden hoffen können, treffen wir auch abg. tïsti, tǐšta, russ. testi, tešča, serb. tast, tašta. Delbrück bemerkt S. 155 dazu, es lasse sich über die Etymologie nichts Sicheres sagen. Darum braucht das Wort natürlich noch nicht jung zu sein. Ich möchte fragen, ob dies nicht mit dem bei Schmeller 1, 583 belegten fränk. tichter 'Enkel' zusammenhängt. Die slavischen Formen weisen auf tiktj, und davon könnte tichter eine Ableitung sein. Schwierigkeiten macht natürlich das $t$. Der Mangel der Überlieferung läßt nicht klar erkennen, ob dies auf urgerm. $\not p$ zurückgehen kann.

Ich fasse also zusammen. Es ist mir durchaus unwahrscheinlich, jedenfalls ist es in keiner Weise zu erweisen, daß bei den Indogermanen die Verwandtschaftsgrade nach der weiblichen Seite nicht bezeichnet worden wären. Wir haben einige Ausdrücke dafür, die indogermanisches Alter haben, andere sind wahrscheinlich verloren gegangen. Zu fragen ist nur, weshalb dies gerade bei den Bezeichnungen für die weibliche Verwandtschaft geschehen ist. Nun in vielen Fällen hat einfach eine Verallgemeinerung der Begriffe stattgefunden, es wird der Ausdruck

1) Anders erklärt Pogatscher das Suffix -inkil, vgl. Anglia 23, $310 \mathrm{ff}$. Dagegen Eckhardt Engl. Stud. 32, 325 ff. und wiederum Pogatscher Anglia Beibl. 1904, 238-247. Pogatscher sieht darin ein wgerm. winkila 'Kind'. Auch diese Deutung würde eninchil tadellos erklären. W. Schulze KZ. 40, 408 sieht in Enkel einfach ein Diminutivum, was ja schließlich auch möglich ist. 
Vaterbruder für Onkel allgemein gebraucht, aber auch umgekehrt. Und daß die Ausdrücke * svekuros ${ }^{*}$ svekr $\bar{u}$ gesiegt haben, ist schließlich nicht wunderbar, da ja die Frau in das Haus des Mannes eintritt. Ich habe früher an die Schrader-Delbrückschen Schlubfolgerungen geglaubt, aber wie man sieht, mit Unrecht. Wundt hat in seinen kritischen Bemerkungen ganz recht.

So erweist sich also auch dieser Punkt, den Schrader für den festesten hielt, als unhaltbar. Die agnatische Struktur der indogerm. Familie folgt nicht aus der Sprache, sondern höchstens aus der Übereinstimmung der Sitten in den ältesten historischen Zeiten.

Aber in diesen zeigt sich eine streng agnatische Ordnung doch nur in sehr modifiziertem Sinne.

\section{E. Götter- und Personennamen.}

Bei diesem Punkte brauche ich mich nicht lange aufzuhalten. Wer den Ausführungen Useners in seinen Götternamen aufmerksam gefolgt ist, dem wird der Grund, weshalb wir verhältnismäßig wenig vergleichbare Götternamen nachweisen können, ganz klar sein. Es gab eben zahlreiche göttliche Gestalten und dementsprechend zahlreiche verschiedene Ausdrücke, und von diesen sind in der einen Sprache die, in der andern die übrig geblieben. Im übrigen sind auch die Gleichungen bei weitem nicht so gering, als es Schrader hinstellt.

\section{F. Sonstiges.}

RL. S. 847 zeigt Schrader, daß die Ausdrücke, die in einzelnen Sprachen 'Tanzen' bedeuten, in andern eine rasche Bewegung ausdrücken. Eine solche Bedeutungsveränderung können wir ja noch heute beobachten, man denke an unser 'drehen, walzen' u. v. a. Wenn wir also Gleichungen haben wie ai. $\jmath^{*}$

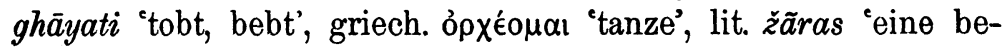
stimmte Art des Gehens', griech. xopóc 'Chortanz, Reigen’ u. a., so folgt für jeden auch nur einigermaßen in der Geschichte der Wörter Bewanderten, daß hier Bedeutungsübergänge allergewöhnlichster Art stattgefunden haben. Da sich aber nicht dasselbe Wort in mehreren Sprachen in der Bedeutung 'tanzen' nachweisen läßt, so schließt Schrader schnell aus dem Negativen folgendes: "Was man aus diesen Tatsachen wird schließen dürfen, ist, daß man in der Urzeit noch kein Bedürfnis empfunden 
haben kann, den Begriff der feierlichen oder leidenschaftlichen Bewegung von dem des Tanzes sprachlich zu unterscheiden, wohl aus dem einfachen Grund, weil man den die Lokomotionsbewegungen zu Tanzbewegungen erhebenden Rhythmus, der sich aus gewissen Arten der ersteren mit Notwendigkeit ergibt, noch nicht als etwas besonderes anzusehen gelernt hatte". Ich konnte diese Worte überhaupt erst gar nicht verstehen, und mußte erst die von Schrader zitierten Ausführungen von Große Anfänge der Kunst S. 213 nachschlagen, um zu erkennen, was der Verf. gemeint haben könnte. Er fährt dann weiter fort: "Tatsächlich müssen auch auf dem Gebiet der Einzelsprachen dieselben Ausdrücke noch lange das Gehen, Hüpfen, Springen und Tanzen bezeichnet haben". Ich kann dem Verfasser verraten, daß diese Sache heute noch nicht aufgehört hat. 'Hüpfen' und 'Springen' werden noch heute im Sinne von Tanzen gebraucht. Vielleicht folgt daraus, daß wir heute "hüpfen, springen und tanzen' noch nicht unterscheiden. "Wie könnte sonst auf römischem Gebiet", ruft der Verfasser emphatisch, "der Name der altehrwürdigen Salier, die doch sicher rhythmisch hüpften von salio und nicht von salto abgeleitet sein?" Ja, wie könnte sonst? daß der Name älter sein kann, als das Aufkommen des Verbums saltare, daß salio ursprünglich 'tanzen' bedeutet haben kann und erst später zu der Bedeutung 'springen' kam, diese Möglichkeiten und andere fallen dem Verf. nicht ein.

Alles in allem halte ich den ganzen Abschnitt für vollständig verfehlt, er zeigt nur, daß sich der Verf. niemals eingehender und genauer mit der Bedeutungsgeschichte einzelner Worte beschäftigt hat.

Genau dieselben Schlüsse finden wir nun unter 'Dichtkunst und Dichter' gezogen (S. 129): "So deutlich der Begriff des gesprochenen Wortes in idg. Gleichungen wie ai. vácas, griech. ËंToc, lat. verbum, got. waúrd hervortritt, umso weniger ausgebildet muß die Terminologie des Gesanges in der idg. Grundsprache gewesen sein". Und weshalb? "Die Bezeichnungen der Einzelsprachen für 'Gesang' sind fast ausschlieBlich aus Wörtern hervorgegangen, welche ursprünglich verschiedene Árten des Sprechens oder Schreiens ausdrücken". Das schließt Schrader daraus, daß derselbe Stamm in der einen Sprache 'singen', in der andern 'schreien, sprechen' bedeutet. Daß die Bedeutung 'sprechen, schreien' ursprünglich ist, liegt in den Tatsachen absolut nicht darin, und es 
ist ebensogut eine Bedeutungsentwicklung von 'singen' zu 'schreien' möglich wie umgekehrt. Man wird oft hören können, er singt nicht mehr, er schreit oder er krächzt, aber daß wir ein Gekrächz ein Singen nennen, ist wohl weniger häufig. Daß man den Hahn den Sänger nennt, ist ganz natürlich. Otfrid sagt tatsächlich thaz huon sang und ebenso sprechen die Südslaven von dem Gesang des Hahnes. Es ist zwar keine Melodie in dem Krähen des Hahnes, wohl aber sind Klänge und wenig Geräusche darin. Ich empfèhle die ganzen Ausführungen RL. auf S. 130 der Beachtung der Wortforscher, sie werden ihre helle Freude daran haben.

$\mathrm{Zu}$ allen diesen Folgerungen kommt aber Schrader nur, weil er aus dem Negativen Schlüsse zieht, und ich glaube nun wohl hinreichend bewiesen zu haben, daß man das besser unterläßt. Es gebricht mir an Zeit, das ganze Reallexikon auf diesen Gesichtspunkt hin durchzugehen. Wer eine Nachlese halten will, wird noch genug finden.

\section{Die partiellen Gleichungen.}

Unter partiellen Gleichungen verstehe ich solche Wortvergleiche, die nur in wenigen Sprachen vorliegen. Der Ausdruck ist $\mathrm{zwar}$ etwas mangelhaft, doch ist er kurz und verständlich und mag daher beibehalten werden. Die partiellen Gleichungen haben durch J. Schmidts Untersuchung über die Verwandtschaftsverhältnisse eine gewisse Berühmtheit erlangt, indem dieser z. T. mit auf sie sein System der Verwandtschaftsverhältnisse gründete.

$\mathrm{DaB}$ Gleichungen nur in zwei Sprachen vorliegen, kann darauf beruhen, erstens daß alle andern Sprachen das betreffende Wort verloren haben. Anderseits werden in allen Sprachperioden neue Wörter gebildet, und, wenn zwei Sprachen eine längere Zeit gemeinsam durchlebt haben, muß sich dies in ihrem Wortschatz zeigen. Da wir aber dies nur für wenige Sprachen annehmen, so kann uns das nicht viel nützen. Drittens aber sind Worte oftmals nur über einen Teil des Sprachgebietes verbreitet, und sie konnten sich dann eben nur in den Sprachen erhalten, die diesem alten Gebiet angehören. Das ist der Sinn, den Joh. Schmidt mit den partiellen Gleichungen verbindet. Ob sich Schmidts Ansicht für die Kulturgeschichte verwerten läßt, ist eine besondere Frage.

Schrader kommt Sprachv. 134 auf etwas zu sprechen, das hierher gehört, und verweist auf die Ausführungen in Kretschmers Einleitung. Kretschmer S. 10 hat darauf Gewicht gelegt, daß wir 
vielfach nicht zu einem einheitlichen Wortschatz der indogermanischen Grundsprache durchdringen können, weil wir für einen Begriff mehrere Gleichungen finden. Er gibt als Beispiel die verschiedenen Zahlworte für 'eins':

\section{ai. $\dot{e} k a-$}

altpers. aiva, aw. aèva-, griech. oifoc 'allein', also in der Bedeutung abweichend.

griech. oivń, l. oinos, altir. oen, got. ains, lit. vênas, abg. inŭ; ai. éna, das vielleicht verwandt ist, bedeutet jedenfalls 'er'.

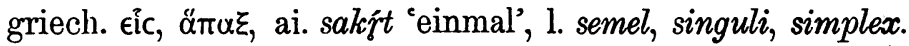

Kretschmer meint dazu S. 12: "In der Zeit, als sich das Bedürfnis nach einer Bezeichnung der Einzahl geltend machte, wurden dafür Wörter von der Bedeutung 'allein', 'zusammen', 'gleich' oder ähnlichen verwendet und zwar setzten sich in den einzelnen Teilen des idg. Gebietes verschiedene Ausdrücke fest". Der Fall, den Kretschmer erörtert hat, steht nicht vereinzelt da, es lassen sich hunderte von Beispielen zusammenbringen, wo eine sprachliche Gleichung nur in einzelnen Sprachen vorhanden ist, während in einem andern Gebiet ein anderes Wort herrscht. Wie sollen wir das erklären? Die Annahme dialektischer Verschiedenheit, wie sie Kretschmer vorschlägt, ist natürlich möglich, sie wird dadurch nahe gelegt, daß noch heute in nahe verwandten Mundarten verschiedene Bezeichnungen derselben Begriffe bestehen. Die Annahme ist also möglich, aber sie ist nicht die einzige und sie ist auch nicht recht wahrscheinlich.

Schrader hat in seinem Reallexikon die Grundsätze aufgestellt, in wie vielen Sprachen und in welchen ein Wort vorhanden sein muß, um es für indogermanisch zu erklären, s. o. S. 57. Nach diesen Grundsätzen müßte er die drei letzten Worte für indogermanisch erklären, und es wird auch keiner daran zweifeln, daß *oiwo, *oino und *sem bis in die Urzeit zurückgehen. Aber sie haben gewiß nicht ein und dieselbe Bedeutung gehabt. Ein Wort für 'eins' muß natürlich vorhanden gewesen sein, denn erstlich kennen wohl alle Völker ein Wort dafür, und zweitens wird man oft genug gezählt haben 1, 2, 21, 22. Insofern ist Kretschmers Satz: "Als sich das Bedürfnis einstellte, die Eins zu bezeichnen", sehr anfechtbar. Dieses Bedürfnis ist gewiß sehr viel älter, als die Zeit, in die wir vordringen können. Daß mehrere Worte für 'eins' bestanden haben, ist nicht glaublich, weil der Sprache der Luxus fremd ist. Hier kommt nun ein 
Gesichtspunkt in Betracht, der durchaus nicht neu ist, und auf den ich in meinen Indogermanen S. 205 erneut hingewiesen habe. Für das, was wir in einem Begriff und einem Wort zusammenfassen, bedürfen einfachere Menschen und Menschen unter andern Lebensverhältnissen mehrerer Worte. Ich brauche hierfür nur an die Jägersprache zu erinnern, die die einzelnen Glieder der einzelnen Tiere, ihre Verrichtungen verschieden benennt, ich brauche nur daran zu erinnern, daß auch bei uns der Mensch ißt und trinkt, das Tier frißt und säuft, daß der Mensch singt, der Hahn kräht, die Nachtigall schlägt. Ganz vorzügliche Bemerkungen über unsern Gegenstand finden sich in dem Buche Unter den Naturvölkern Zentral-Brasiliens von K. von den Steinen.

Ich habe die Stelle schon in meinen Indogermanen abgedruckt und will sie hier nicht wiederholen. K. v. d. Steinen weist darauf hin, daß bei primitiven Völkern viel weniger allgemeine Zusammenfassungen angewendet werden als auf Stufen höherer Entwicklung. Schrader hätte aber die wesentlichen Tatsachen schon aus Osthoff Vom Suppletivwesen in den indogerm. Sprachen, Heidelberg 1899 entnehmen können; dort wird S. 79 auf Jespersen progress in language hingewiesen, wo reiches Material $\mathrm{zu}$ finden ist. Die Ureinwohner von Tasmanien hatten keine Wörter, um die sogenannten 'general terms' sprachlich darzustellen, also z. B. kein Äquivalent für den Ausdruck Baum, anderseits dagegen je einen besondern Namen für jede Spielart des blauen Gummibaums, der Akazie und anderer Baumund Pflanzengattungen. Die Gesellschaftsinsulaner reden von einem Hundeschwanz, dem Schwanz eines Schafes und dergl. mehr, der Schwanz an sich aber ist etwas ihnen und ihrer Sprache Fremdes. Die Mundarten der Zulu weiß wohl eine 'rote', eine 'weiße', eine 'braune' Kuh, nicht jedoch eine Kuh im allgemeinen $\mathrm{zu}$ bezeichnen usw.

$\mathrm{Daß}$ wir ähnliches auch für die Indogermanen vorauszusetzen haben, habe ich meinen Indogermanen verschiedentlich ausgeführt, nachdem Joh. Schmidt die Berechtigung des Standpunktes für das Litauische gezeigt hatte, Kritik der Sonantentheorie S. 37.

$\mathrm{Ja}$, wir haben in unserer Sprache selbst zahlreiche ganz ähnliche Fälle. Die Jägersprache benennt dieselben Glieder verschiedener Tiere mit verschiedenen Namen. Der Hirsch hat Läufe, 
das Schwein Hammen, der Bär Tatzen, der Wolf Klauen. Oder man denke an die verschiedenen Ausdrücke für Junge werfen. Und reden wir nicht auch von einem Schimmel, einem Rappen, einem Fuchs.

Osthoff hat auch schon a. a. O. S. $47 \mathrm{ff}$. die verschiedenen Ausdrücke für 'eins' zu erklären versucht. $O b$ er im einzelnen recht hat, tut nichts zur Sache: der Grundgedanke ist sicher richtig. Ich stimme in der Erklärung ganz J. Schmidt KZ. 36, 397 bei. 'sem bezeichnet eigentlich die 'Eins', die aus der Vielheit entsteht, 'oinos ist einer unter mehreren', oiwos bedeutet 'allein'. Schließlich haben auch wir für diese verschiedenen Begriffe noch besondere Worte, wenn auch anderseits unser ein sehr verschiedenerlei bezeichnen kann. Man vergleiche zum folgenden Grimm DWB. s. v. Wir finden ein 1) in der Bedeutung 'einer' unter mehreren, z. B. 'ein und zwanzig'; 2) in der Bedeutung der Vereinigung; sie werden ein Fleisch sein, alles war ein Herz und eine Liebe, es ist alles ein Feuer, 3) in der Bedeutung 'allein', solus, $\mu$ óvoc, oioc, mhd. wir zwei beliben eine Iw. 331, er reit al ein gein wunders not Parz. 432, 30. Außerdem gibt es noch eine ganze Reihe verschiedener Bedeutungen. Für die zuletzt aufgeführten brauchen wir heute wieder 'allein'. Aus alledem folgt, daß wir heute vielfach einen Ausdruck anwenden, wo die ältere Sprache mehrere gebraucht hat, und daß es daher sehr wichtig ist, bei mehreren konkurrierenden Worten die genaue Bedeutung festzustellen. Es ist ganz klar, daß durch diese Auffassung bei der Erschließung des idg. Wortschatzes manches anders wird aufgefaßt werden müssen als früher. Von alle dem bietet aber Schrader seinen Lesern nichts, obgleich der Gesichtspunkt nichts weniger als neu ist. Es folgt aus der oben skizzierten Grundroraussetzung ferner, daß sich gerade die Ausdrücke für die Gegenstände der materiellen Kultur immer mehr verringern werden. Es läßt sich scheinbar für viele sicher bekannte Gegenstände kein Ausdruck nachweisen, d. h. es liegt kein Wort mit der gleichen Bedeutung in mehreren Sprachen vor. Das wird in vielen Fällen so zu deuten sein, daß eine Vielheit von Ausdrücken für diesen Gegenstand vorhanden war. Jedenfalls brauchen die partiellen Gleichungen, d. h. solche, die uns nur in zwei oder drei Sprachen entgegentreten und mit denen Schrader immer wieder operiert, gar keine besondere Bedeutung zu haben. Sie können, ja sie müssen ebenso gut indogermanisch sein, wie andere 
weiter verbreitete. Jedenfalls darf man die partiellen Gleichungen nicht bald so, bald so verwenden.

Wir haben z. B. eine Fülle von Gleichungen für 'Milch', die sich, wie Schrader bemerkt, merkw ürdigerweise immer auf zwei Sprachen beschränken, ja zum größten Teil, wie man hinzufügen muß, auf einander ziemlich nahestehende Sprachen: ai. dadhan-, altpreuß..dadan; griech. ráda, lat. lac; got. miluks, ir. melg; ai. ghrtá- 'Butter' und ir. gert 'Milch' stimmen zwar formal, aber nicht genau in der Bedeutung.

Würde Schrader diese Gleichungen beurteilen, wie die Ackerbaugleichungen, so würde er $7 u$ dem Schluß kommen müssen, $\mathrm{da}$ die Milch wahrscheinlich nicht verwendet wurde. Ich glaube, auch hier wird es sich um Worte handeln, die ursprünglich etwas verschiedenes bedeuten. dádhi heißt es in Indischen 'saure Milch'. Außerdem kann man die einzelnen Milcharten 'Schaf-, Ziegen-, Kuhmilch' durch besondere Worte unterschieden haben, wie wir das durch unsere Komposita bewirken ${ }^{1}$ ).

Dasselbe gilt nun für die mannigfach wechselnden Benennungen für die einzelnen Tiere, für die Farbenbezeichnungen und vieles andere. Auf die verschiedenen Bezeichnungen der Hand habe ich schon in meinen Indogermanen hingewiesen. Einerseits erklären sich diese, daß verschiedene Worte einmal einen besonderen Teil der Hand bezeichneten, griech. ỏroctóc 'flache Hand',

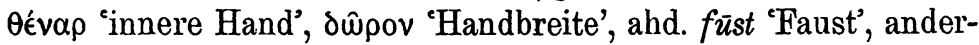
seits können auch an verschiedenen Orten verschiedene Worte vorhanden gewesen sein.

Auch für 'Vater' gibt es mehrere Ausdrücke. Ai. tatá hat sicher so viel Anrecht, der idg. Grundsprache zugeschrieben zu

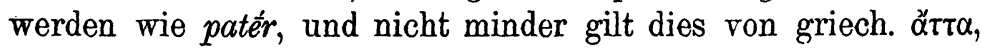
got. atta, abg. otič.

Wenn Delbrück Verwandtschaftsnamen 452 sagt: "Ein besonderes Wort für Eltern scheint im Idg. nicht vorhanden ge-

1) Lat. lac und griech. $\curlyvee \alpha \dot{\lambda} \alpha \alpha$ gehören trotz der lautlichen Schwierigkeiten, über die man Stolz IF. 14, 20 vergleiche, zusammen. Meines Erachtens lösen sich alle Schwierigkeiten unter dem Ansatz von idg. $d l$. al wurde im Lat. $z u l$, im Griech. zu $g l$, vgl. ridroc, von dem aus das $\gamma$ auf $* \delta \alpha \lambda \alpha$ übertragen wurde, vgl. dulcis und $\gamma \lambda u \kappa u ́ c$ Für das germanische Wort setze ich ebenfalls *delag voraus, dessen Anlaut durch die Wörter 'Melken, Molken' umgestaltet wurde. Auch alb. đala kann hierhergehören; man braucht es aber nicht mit G. Meyer auf gal zurückzuführen, vgl. đaš “ich gab". 
wesen zu sein", so ist das möglicherweise richtig und braucht nicht beanstandet zu werden. Besser wäre es zu sagen, ein besonderes Wort für Eltern können wir nicht nachweisen. Wenn aber Schrader RL. 182 bemerkt: " Wabrscheinlich war eine Bezeichnung für Eltern in der Urzeit überhaupt nicht vorhanden, da die ganz verschiedenartige Stellung, welche Vater und Mutter den Kindern gegenüber einnahmen, die Ausbildung einer zusammenfassenden Bezeichnung für dieselben verhindern mochte", so ist das Phantasterei und ein absolut unzulässiger Schluß. Man braucht, wie schon Delbrück S. 452 bemerkt, in verschiedenen Sprachen den Plural (ursprünglich wohl den Dual) von Vater, und das kann schon indogermanisch sein. Daneben können Wörter, die

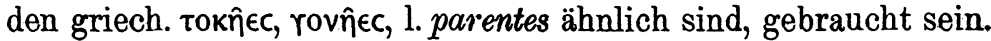
Man beachte auch, daß jetzt schon weite Kreise für 'Eltern' einen neuen Ausdruck 'die Alten' geprägt haben.

Steinens Buch hätte auch bei den Erörterungen über die Farbenbezeichnungen gute Dienste geleistet. Auch Schrader möchte glauben ${ }^{1}$ ), daß von einer Farbenblindheit der Indogermanen keine Rede sein kann. Warum wieder so zaghaft? Wir können zahlreiche Farbenausdrücke für das Idg. nachweisen, also kann von Farbenblindheit keine Rede sein.

Wir finden dann wieder die Bemerkung, daß eigentlich nur eine Farbe, nämlich das Rot, bei allen oder nahezu allen Indogermanen dieselbe feste Bezeichnung hat. Aber nach Schraders oben ausgesprochenen Prinzipien müssen wir folgende Ausdrücke für idg. halten :

Lat. helvus, ahd. gelo, ai. hárita-, av. zairita, abg. żlütüu, lit. geltas; ai. hariná, abg. zelenǔ; ai. malinas, griech. $\mu \in \lambda^{\prime} \lambda \alpha c$; griech.

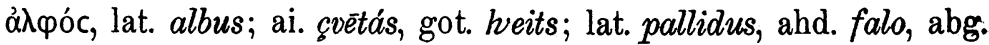
plavú u. v. a. Daß die Ausdrücke manchmal in der Bedeutung variieren, kann unmöglich etwas zur Sache tun. Die Erhaltung des Ausdruckes 'rot' in fast allen Sprachen aber ist jedenfalls auf die besondere Stellung, die das Rot überall einnimmt, zurückzuführen.

Es ist meines Erachtens also durchaus möglich, daß die partiellen Gleichungen darauf zurückgehen, daß von mehrere Ausdrücken, die verschiedene Seiten eines Gegenstandes oder einer Tätigkeit bezeichneten, der eine hier, der andere dort verloren gegangen ist, weil man in Laufe der Zeit die besondern Seiten

1) Von mir gesperrt. 
94 H. Hirt, Untersuchungen zur indogermanischen Altertumskunde.

nicht mehr hervorhob. Ich möchte hierfür noch ein Beispiel anführen. Für uns Stadtleute ist es von Bedeutung, ob es regnet oder nicht regnet, ob man also einen Schirm braucht oder nicht. Ganz anders ist es auf dem Lande, da macht man eine Fülle von Unterschieden. J. H. Campe sagt in seinem Wörterbuch zur Erklärung und Verdeutschung der fremden Ausdrücke, Braunschweig 1813 S. 57: "Für die verschiedenen Abstufungen des schwächern oder stärkern, des feinern oder gröbern Regens, kann ich, nach einem kurzen Besinnen, acht niederdeutsche Stufenwörter aus dem Gedächtnisse angeben; sehr möglich, oder vielmehr sehr wahrscheinlich, daß es deren noch eine größere Anzahl gibt. Es sind: 1) es mistet, von dem feinsten Staubregen; 2) es schmuddert, d.i.: es regnet ein wenig und fein; 3) es stippert, d. i. es fallen einzelne und zwar gleichfalls feine Regentropfen, die aber doch schon etwas größer als bei dem Misten und Schmuddern gedacht werden; 4) es regnet; 5) es pladdert, d.i. es regnet stark und laut; 6) es guddert, wodurch das Geräusch des bei einem sehr starken Regen von den Dächern herabströmenden Wassers ausgedrückt wird; 7) es gießt, und 8) es gießt mit Mollen, für den stärksten Grad des Platzregens".

Campe gibt auch noch anderes Material, das hierher gehört. So hat das Ndd. einen besonderen Ausdruck für 'schnell laufen', nämlich kleppen, wovon Klepper. "Ebenso hat er nicht bloß für den Begriff des stärkern Eilens hasten, sondern auch für den höchsten Grad desselben, welcher mit Verwirrung und Unordnung verbunden zu sein pflegt, das sehr ausdrucksvolle Wort hasterbastern". Schließlich gibt es ja auch für das Wehen des Windes, vom sanften Wehen bis zum Sturm eine Fülle von abstufenden Bezeichnungen.

Wir wollen aber auf den Regen zurückkommen. Schrader sagt: " Eine indogermanische Bezeichnung hierfür liegt in ai. varș̆á-, ir. frass, griech. 'epcn (letzteres 'Tau'). Sonst gehen die Namen auseinander". Sicher ist also das, was wir für die indogermanische Grundsprache erschließen können, außerordentlich dürftig, und es ist wohl ganz sicher, daß die Indogermanen über eine Fülle von Ausdrücken verfügt haben. So bedeutet lit. lytùs eigentlich den 'Guß', lat. pluit zu ahd. fliozzan vielleicht etwas ähnliches, und deutsch regen wird wieder eine besondere Art des Regens bezeichnet haben.

Für Nebel haben wir auch zwei Ausdrücke griech. ỏ $\mu i x \lambda \eta$, 
abg. miggla, und lat. nebula, d. nebel (letzteren Ausdruck vergißt Schrader merkwürdigerweise), und auch hier werden ursprünglich verschiedene Bedeutungen anzunehmen sein.

Ich denke, es ist nun klar, daß die partiellen Gleichungen keineswegs mit Notwendigkeit oder auch mit Wahrscheinlichkeit auf dialektische Verschiedenheiten innerhalb derindogermanischen Grundsprache hinweisen, sondern daß sie ebenso gut gedeutet werden können, wenn wir annehmen, daß eine Fülle besonderer Unterscheidungen bestand, die die Sprachen im Verlauf der höhern Kulturentwicklung nicht beibehielten. Wenn also Schrader 9 Gleichungen anführt, die eine andere Bewegung als das Gehen ausdrücken, so wird jede dieser Gleichungen eine besondere Nuance ausgedrückt haben. Man bedenke, wie viel verschiedene Ausdrücke wir noch haben: schleichen (langsam gehen), schlendern gehen, schreiten, trippeln, stolzieren, marschieren, springen, laufen, eilen, hasten, hasterbastern, hüpfen, stürmen, rennen, rasen, und wir tanzen nicht nur, sondern wir walzen, wir galoppieren, die jungen Mädchen hüpfen wie die Lämmer, er tanzt wie ein Bär, wir schwofen, wir drehen uns u. a.

Diesen z weifellos wichtigen Gesichtspunktkennt nun Schrader gar nicht, obgleich er längst bekannt war, und es ist deshalb seine ganze Betrachtung der sprachlichen Tatsachen nicht zutreffend. Die Betrachtungsweise der ältern Forschung, die einfach die sprachlichen Tatsachen an einander reihte, ist im Grunde viel besser, weil sie eben den sprachlichen Tatsachen keine Gewalt antat.

[Fortsetzung folgt.]

Leipzig-Gohlis.

H. Hirt.

\section{Zu den arischen Wörtern für 'der erste' und 'der zweite'.}

\section{A. primus und prior im Arischen.}

1. Ob die altarischen Dialekte die Begriffe 'der erste' von mehreren und 'der erste' von zweien durch dieselben sprachlichen Mittel zum Ausdruck gebracht haben oder durch verschiedene wie das Lateinische durch primus und prior, darüber geben unsre Grammatiken, soweit ich sehe, keine Auskunft. In der Tat hat eine solche Unterscheidung bestanden, und es sind zur Darstellung der beiden Begriffe je mehrere Wörter gebraucht 